\title{
Work In Progress (WIP): A Systematic Review Describing Impacts on Engineering Undergraduates who Participate in Outreach
}

\author{
Dr. Joanna K. Garner, Old Dominion University \\ Dr. Garner is Executive Director of The Center for Educational Partnerships at Old Dominion University, \\ VA.
}

\section{Prof. Karen A. Thole, Pennsylvania State University, University Park}

Karen A. Thole serves as the head of the Department of Mechanical Engineering at Pennsylvania State University and also holds the title of Distinguished Professor. She is the co-founder of the Engineering Ambassador Network, which is a professional development program for engineering students with an outreach mission to high school students. Her area of expertise is turbine cooling and using additive manufacturing to develop innovative cooling technologies. She has published over 220 archival papers and advised 70 dissertations and theses. Dr. Thole has provided service leadership to numerous organizations including being a member of ASME's Board of Governors. She has also served as the Chair of the Board of Directors for the ASME's International Gas Turbine Institute in which she led a number of initiatives to promote communities of women engineers and students. In her roles as an educator, researcher, and mentor, Dr. Thole has received numerous awards. The most notable awards include, being recognized by the United States White House as a Champion of Change for STEM. She has been recognized by Penn State's Rosemary Schraer Mentoring Award and Howard B. Palmer Faculty Mentoring Award. She was recognized in 2014 by the Society of Women Engineers' Distinguished Engineering Educator Award and in 2016 by ASME's Edwin F. Church Medal ASME's George Westinghouse Medal. In 2017, she received ABET's Claire L. Felbinger Award for her work in diversifying engineering. In 2019, she received AIAA's Air Breathing Propulsion Award for her contributions to promoting diversity and for her technical work in gas turbine cooling.

Dr. Thole holds two degrees in Mechanical Engineering from the University of Illinois at UrbanaChampaign and a PhD from the University of Texas at Austin.

\section{Mr. Michael Alley, Pennsylvania State University, University Park}

Michael Alley is a professor of teaching for engineering communications at Pennsylvania State University. He is the author of The Craft of Scientific Writing (Springer, 2018) and The Craft of Scientific Presentations (Springer-Verlag, 2013). He is also founder of the popular websites Writing as an Engineer or Scientist (www.craftofscientificwriting.com) and the Assertion-Evidence Approach (www.assertionevidence.com). 


\section{Work In Progress (WIP): A Systematic Review Describing Impacts on Engineering Undergraduates Who Participate in Outreach}

\section{Overview}

To teach STEM content to K-12 students, and recruit talented and diverse K-12 students into STEM, many outreach programs at universities in the United States rely on the collective efforts of undergraduate students. Outreach design and research have typically focused on the K-12 students and their teachers, leaving the effect of the outreach on the STEM undergraduates themselves to be an important but overlooked consideration that has received less attention in the engineering education literature. This WIP paper describes the preliminary findings of a systematic review of literature on the impact(s) of involving undergraduates in engineering outreach with a particular focus on studies that report on the impact on the undergraduate students. Supporting this effort is the NSF EArly-concept Grant for Exploratory Research (EAGER) program.

\section{Introduction}

In response to the need to increase interest and persistence in STEM careers, many universities have created organized outreach initiatives. Engineering outreach by undergraduate students takes different forms but can include leading summer camps, teaching afterschool programs, conducting classroom presentations, and hosting engineering fairs and competitions on colleges campuses. The focus of evaluation efforts for K-12 outreach programs is typically on the ways in which students and teachers are impacted, but participation in K-12 outreach has also been portrayed as a means of improving undergraduate students' affective and motivational wellbeing, including their engagement, persistence, and personal identification with a subject area such as engineering [1]. Some scholars have argued that outreach participation, like other forms of co-curricular activity, provides students with a sense of community and belonging, increases resilience, and minimizes feelings of marginalization [2]. Others have emphasized that outreach participation can act as an opportunity for undergraduate students to develop critically important professional skills that might not otherwise be learned in typical undergraduate courses such as technical communication, teamwork, leadership, and the ability to integrate across engineering disciplines [3-4, 52]. In this systematic review of literature, we sought to better understand affective and cognitive facets of outreach impact and draw conclusions about the overall nature of research conducted in this area.

We used systematic review methodology to guide this work [5]. Systematic reviews treat individual studies as sources of data and involve a series of steps that are guided by research questions. After defining and executing a number of inclusion and exclusion criteria for the set of papers to be considered, common features of studies are extracted and compared with one another. Findings are typically reported using tables that organize the critical features of each study as well as via a narrative form that describes overall trends or findings. In this case, we used an aggregative synthesis approach, which is typically used to investigate questions about impacts or effects and reveal descriptive information about the state of the field from which directions for future research can be derived. Our three research questions were as follows: 
1. What research designs are used to examine the impact of outreach on undergraduate engineering students?

2. What theories are used to examine the impact of outreach on undergraduate engineering students?

3. What themes emerge from studies' findings about the impact of outreach on undergraduates?

\section{Method}

The first steps were to develop inclusion and exclusion criteria for the review. Criteria for including the papers were fourfold: (1) describe programmatic outreach efforts from one or more colleges of engineering to K-12 audiences, (2) include undergraduate engineering students as ambassadors or mentors, (3) take place within the continental United States, and (4) include evidence of impacts on the undergraduate students. In other words, papers were excluded if they (1) described undergraduate involvement in service learning, affinity group, or community engagement projects, (2) included undergraduates simply as chaperones or creators of classroom activities, (3) described activities undertaken in overseas settings, and (4) did not report any impacts on undergraduate students conducting the outreach. Next, search terms were defined to cover our areas of interest. Three academic databases were searched using all three terms: engineering + undergraduate students + outreach. Records were retrieved for 1996-2018. The ASEE search revealed 3,403 records, and the ERIC and Education Research Complete searches revealed just 23 and 7 records, respectively. Using the search terms in a historical manner revealed a significant upward trend in the number of papers describing projects and research studies that involved undergraduate students in an outreach capacity. For example, whereas a search of the 1996 ASEE PEER database yielded just 14 papers, this number had grown to 124 by 2006 and 303 by 2016 .

The large corpus of literature became much smaller once three of the four complementary inclusion and exclusion criteria were applied. Only 462 papers were eligible for an informational value assessment, which involved detailed reading and an examination of the quality of evidence provided under the fourth inclusion/exclusion criterion. Once again, a historical trend was evident; for example, only 3 papers being retained from the 1996 PEER archive, compared with 14 in 2006 and 82 in 2016. When examined using the fourth inclusion criteria, 48 papers were found to include primary qualitative or quantitative data from which claims were supported. These papers were then subject to critical feature extraction.

Several features of each paper were recorded. These included the authors of the paper and its year of publication, the institution(s) at which the outreach was taking place, the name of the outreach program, the outreach model and a description of any professional development or training received by undergraduates, the program's premise or underlying theory of action, the study purpose or research questions that guided the paper, the methods used to investigate impact on undergraduates, and the main findings in relation to the impact on undergraduates. Additional details about impacts on K-12 students or teachers were not recorded.

\section{Findings}

The critical features of each reviewed study are summarized in Tables $1 \mathrm{a}$ and $1 \mathrm{~b}$, which are organized by research approach. Studies investigating change over time are presented in Table 1a. Studies utilizing post-hoc responses are included in Table $1 \mathrm{~b}$. 


\section{Research Question 1. What research designs are used to examine the impact of outreach on undergraduate engineering students?}

The two most common research designs included surveys of undergraduates in either a single-group pre-test/posttest design $(n=10)$ or a posttest only design $(n=31)$. Only one study included a comparison group in which outreach participation was compared with a comparison group of students who participated in an introductory engineering course instead. Most $(n=31)$ of the studies relied on a survey with either Likert scale or open-ended questions as a form of data collection. Just two studies explicitly referred to the use of previously validated instruments. Five studies took a qualitative interview or diary approach. Several studies exclusively used non-selfreport data sources including outreach logs, work products, observations, or undergraduate student retention data.

\section{Research Question 2. What theories are used to examine the impact of outreach on undergraduate engineering students?}

Since most of the papers did not implement previously validated measures, and very few included surveys or interview questions as appendices, we drew from the background sections of the papers to better understand the constructs and theoretical frameworks researchers used to shape their understanding of the impact of conducting outreach on the undergraduates. Most of the papers included a brief literature review, but only a small number of papers included an explicit theoretical perspective to frame how and why outreach might impact undergraduates. Many papers referred to general concepts such as peer mentorship, cohort-based learning, or communication and professional skills, but did not operationally define them in relation to explicit research questions or specific measures. However, in studies where this was apparent, we noted that one of four salient theoretical perspectives was evoked: (1) Valence, Instrumentality, Expectancy theory [6], (2) self-efficacy [7-8], (3) extra-curricular learning [9], and (4) social role identity theory [10-11].

(1) Valence, Instrumentality, Expectancy theory (VIE) [6] was used in several studies as a framework for understanding undergraduate students' motivation to participate in engineering outreach. This theory proposes that motivation arises from the interaction among elements of valence, or value of an activity, instrumentality, or the connection between an activity and the individual's goals, and expectancy, or the individual's perception that the activity can be successfully performed. Switzer and Benson [12] used VIE theory to examine changes in undergraduate engineering students' motivation as a result of participating in a three-week period of outreach. The premise of the study was that motivation would be impacted by outreach, and that motivation is also linked to remaining in an undergraduate engineering program. Although they did not find statistically significant changes, they noted positive shifts in students' responses to questions about value, instrumentality and expectancy of their engineering studies. Atwood \& Fry [13] used the VIE framework to guide the development of open-ended prompts given to undergraduate engineering students after they had completed an outreach event. Using descriptive statistics and thematic analyses, the authors reported gender differences in how men and women viewed the outreach activity. Whereas valence was highly represented in men's responses, expectancy was a more prominent theme for women. Thematic differences were also apparent in the instrumentality of the activity, with women more likely to record goals of exciting students about engineering and men more likely to articulate goals of teaching content. 
Bigelow [14] also used a VIE-informed reflection paper to investigate undergraduate engineering students' motivation towards outreach after participating in a biomedical engineering course in which an outreach activity was included. Using an inductive coding process, Bigelow identified 12 themes within the reflections, but these focused on lessons learned rather than valence, instrumentality, and expectancy specifically. Themes pertaining to shifts in undergraduates' assumptions or expectations included their recognition of the importance of hands-on learning, their surprise at how engaged the K-12 students were, and their discovery that young students did not know what engineering was. In addition, the undergraduates seemed divided on "whose job" it is to increase awareness of engineering as a career field, suggesting that they began to consider the broader societal issue of how to increase participation in engineering.

(2) Self-efficacy, defined as one's perception of one's own capability to successfully complete a task [7-8] was used as a guiding framework for assessing impact on ambassadors in a study by Anagnos, Lyman-Holt, Marin-Artieda and Momsen [15]. The study was grounded in the premise that ambassadors would benefit from self-efficacy for outreach and its associated components, including engineering knowledge and professional communication skills. Most of the 51 ambassadors responded that they perceived gains in skills to motivate others, resolve interpersonal conflicts, adjust when things were not going to plan, manage time, engage in teamwork, explain technical concepts, and speak in front of an audience. Ambassadors' confidence in their abilities was also high as a result of participating in outreach. Longer time in the ambassador program and more senior roles were associated with high rates of student agreement that they had experienced changes in confidence in a number of areas, including confidence to effect positive change through leadership, confidence to succeed in engineering, confidence to speak in front of others, and plans to attend graduate school.

Greene, Zhan, Anthony, Post \& Parkhurst [16] used self-efficacy to consider the impact of outreach on teachers and undergraduate engineering students. In addition to interviews and field notes, the researchers administered the Teachers' Sense of Self-Efficacy Scale to teachers and undergraduate engineering students before and after they had implemented an outreach activity for middle school students. They found statistically significant gains in self-efficacy to engage students in outreach, which included being able to motivate students and help them value learning about engineering.

(3) The benefits of extra-curricular learning for undergraduate engineering students participating in outreach were explore in by Yowell, Zarske, Knight, and Sullivan [8]. Yowell et al. drew from prior work by Astin \& Sax [9], who found that students who participated in volunteer extra-curricular activities, including outreach, reported an increased sense of civic responsibility and life skills. In addition, Yowell et al hypothesized that women might benefit more than men from engaging in service activities due to the provision of real-world connections and pro-social aims of the application of technical content. The researchers surveyed over 100 current and former undergraduate engineering peer mentors who had led after school engineering and STEM clubs in local elementary schools and asked about perceived impacts on professional and career skills including oral communication skills, fundamental engineering skills, future career plans, satisfaction with engineering studies, and undergraduate course selections. There were two areas where responses were nearly unanimous: most $(85 \%)$ of the undergraduates currently in the leader role agreed that participation had impacted their oral communication skills, and $75 \%$ agreed that it had impacted their satisfaction with their engineering studies. 
(4) Recently, researchers have begun to examine the impact of participating in outreach through the lens of identity formation and change, using frameworks based in social identity theory (SIT; [10]) and role identity theory [11]. Ross, Fletcher, Thamotharan \& Garcia [17] examined the impact of a mentorship model of outreach by asking undergraduates to reflect on their self-perceptions. They drew from Godwin, Potvin, Hazari \& Lock [18], who disaggregated the components of an engineering identity into performance/ competence, defined as a person's perception of their own competence in a field; interest, defined as perceived interest; and, recognition, defined as the degree to which the person perceives that others define or recognize them in a particular role. The researchers examined the impact of outreach on the ways that participants might talk about engineering, might act in or otherwise participate in engineering, might describe themselves in relation to engineering and how they might relate to others in the community in relation to engineering. A combination of inductive and deductive coding revealed that all but one of the respondents saw themselves as a "math, science, computer science, or engineering person." They expressed an identification with their respective STEM field and that participating in outreach had increased their awareness that others saw them as a "STEM" person and that acting in this role could bring altruistic gains in terms of inspiring others to follow a STEM career.

Other research used the Dynamic Systems Model of Role Identity [11] to examine the impact of engineering ambassador participation on undergraduates' developing sense of identity. The researchers propose that although students may possess multiple social roles, the role of ambassador can impact other roles such as young professional or beginning engineer. In several multiple case studies, Garner and colleagues $[4,19]$ interviewed newly trained and experienced engineering ambassadors, and revealed alignments between the students' personal histories and interests with engineering and the goals of the outreach program in which they were involved. Students' existing values and beliefs were found to align with the solution messages in the National Academy of Engineering Changing the Conversation report. Outreach training, mentorship and coordination activities allowed students to incorporate and practice new skills in technical communication and leadership in their roles as an ambassador and student leader, with several students recounting that such experiences also benefit their academic performance.

\section{Research Question 3. What themes emerge from studies' findings about the impact of outreach on undergraduates?}

An inductive, thematic analysis of the studies' main findings suggests two main clusters of impact on undergraduate engineering students. The first cluster can be defined as perceived changes in students' technical skills and career preparation, and the second cluster reflects impacts on students' identities and motivation.

Technical skills and career preparation. The majority (65\%) of studies reported impacts on the undergraduate students' communication and/or presentation skills. Some studies also reported impacts on students' perceptions of their teamwork and organizational skills, time management skills, and their ability to present technical information to non-technical audiences. None of the studies we examined collected data pertaining to students' ordinary academic and coursework experiences. Therefore, the degree to which these skills actually transfer for the benefit of course achievement remains unknown.

Identities and motivation. A smaller subgroup (35\%) of studies reported impacts of outreach participation on undergraduate students' motivation and identity. In regard to 
motivational constructs, outreach participation was found to influence students' interest in continuing to engage in outreach efforts in the future, and was associated with increased satisfaction with engineering as a degree. Outreach participation was also associated with undergraduate agreement with the statement that they perceive themselves as a "STEM" person, which suggests that outreach can be linked, although not causally, to the development of a personal identification with the subject area. Intersectionality was noted in some studies in regard to gender, race, and demographic identities. Some studies reported that outreach led students to recognize the need and value of working with particular groups of underserved students, while others highlighted the ways in which students engaged in outreach might be motivated to do so due to personal affinities with underserved groups.

\section{Discussion}

This Work in Progress paper reports on the preliminary findings of a systematic review of studies investigating the impact of outreach participation on undergraduate engineering students. Our review indicates that outreach impacts students' technical and career preparation skills, specifically in the areas of communication and leadership, by providing explicit training and specific opportunities to convey engineering concepts to non-technical audiences. Outreach participation also has motivational and identity-related impacts on students by enabling them to explore new engineering-related roles in professional contexts and leverage their existing beliefs in pro-social applications of the field. Participation as an outreach mentor was linked to improved retention in the engineering degree and high levels of satisfaction with the choice to undertake an engineering degree.

Despite these promising findings, our review reveals areas where further research is needed. The research designs used to examine the impact of conducting outreach typically focused on evaluating the outcome for a single cohort of undergraduates, or changes in the knowledge and skills of undergraduates. This means that it is not possible to state the relative benefit or impact compared to students who do not participate in outreach.

Some studies described a professional development model used to develop the students' outreach skills, while others did not. Definitions of training also differed from one outreach model to another; student outreach preparation varied from participation in a course in which an outreach design assignment was included to required coursework in technical communication and weekly meetings to strengthen students' skills. Because very few studies connected specific aspects of professional development to particular outcomes, and none of the studies compared professional development models to one another, the question of which models of professional development might be optimal for which students in which contexts remains open.

A second area in which an empirical and methodological need emerged was in the measures or indicators of impact on the undergraduate students. Studies often included a researcher- or evaluator-designed survey or interview protocol. Although this approach might allow the investigators to ask questions specific to particular outreach programs or experiences, it made it challenging to compare findings across programs. In future, the development and validation of a uniform instrument to measure the impact of conducting outreach would allow researchers to investigate the impact of various types of experiences of different degrees of involvement on particular skills, attitudes, and behaviors. Similarly, the research designs used to investigate the impact of outreach are often descriptive rather than causal in nature. More research is needed to examine the effect of outreach as an intervention for a broad range of 
undergraduate students rather than a pre-selected, high ability or minority-only subset of students. Longitudinal quantitative or qualitative research might prove to be effective in revealing the medium- and long-term impacts on students' post-graduation outcomes.

Finally, this review uncovered a need for more systematic application of theories and frameworks that can help researchers and evaluators synthesize existing knowledge about outreach participation, as well as formulate and investigate new questions about the impact of engineering outreach on undergraduates. The studies included in the review used a variety of constructs and models to conceptualize the impact of conducting outreach. Many studies did not draw a clear line between theoretical constructs and the measurement of outreach impact. A more comprehensive picture might be gained from studies that integrate theories of student engagement, college student learning, motivation, and development, and identity formation, and apply them in such a way as to acknowledge the intersectionality between various forms of social and personal identity and the experience of conducting engineering outreach. Next steps for our research group include a more detailed synthesis of these frameworks and the development and validation of a measure that can be used across different outreach programs.

\section{Conclusion}

The current body of literature suggests the presence of common impacts on undergraduate engineering students who participate in outreach. Communication and technical skills were frequently included as an area of improvement, along with motivational and identityrelated constructs such as identification with engineering and self-efficacy for professional behaviors. Although research and evaluation of engineering outreach has increased over the past 20 years, further efforts must more clearly theorize, assess, and compare the impact of various types and intensities of outreach participation.

\section{Acknowledgments}

We wish to thank the National Science Foundation EARly-concept Grant for Exploratory Research (EAGER) program for their provision of award 1752096 in support of this work. 
Table 1a. Critical Features of Studies Examining Impact Pre-and Post-Outreach

\begin{tabular}{|c|c|c|c|c|c|c|c|}
\hline $\begin{array}{l}\text { Authors } \\
\text { and } \\
\text { Citation } \\
\text { Number }\end{array}$ & Institution & Program & $\begin{array}{l}\text { Outreach } \\
\text { Model }\end{array}$ & $\begin{array}{l}\text { Premise or } \\
\text { Theory of Action }\end{array}$ & $\begin{array}{l}\text { Purpose or } \\
\text { Research } \\
\text { Questions }\end{array}$ & Methods & $\begin{array}{l}\text { Impact on } \\
\text { Undergraduates } \\
\text { Conducting Outreach }\end{array}$ \\
\hline $\begin{array}{l}{[20]} \\
\text { Thole, Zappe, } \\
\text { Marshall, Alley } \\
\text { \& Engel (2013) }\end{array}$ & $\begin{array}{l}\text { Pennsylvania } \\
\text { State } \\
\text { University }\end{array}$ & $\begin{array}{l}\text { Engineering } \\
\text { Ambassadors } \\
\text { Network (EAN) }\end{array}$ & $\begin{array}{l}\text { Three characteristics } \\
\text { of EA program: } 1 . \\
\text { Communication } \\
\text { training workshop; } 2 \text {. } \\
\text { Outreach performed } \\
\text { in middle and high } \\
\text { schools; } 3 \text {. Academic } \\
\text { coursework to } \\
\text { develop professional } \\
\text { skills. }\end{array}$ & $\begin{array}{l}\text { Outreach provides a } \\
\text { professional development } \\
\text { vehicle for engineering } \\
\text { undergraduates. }\end{array}$ & $\begin{array}{l}\text { Impact of training; } \\
\text { process of scaling } \\
\text { the network. }\end{array}$ & $\begin{array}{l}\text { Pre- and post- } \\
\text { training } \\
\text { workshop } \\
\text { surveys of } 22 \\
\text { undergraduate } \\
\text { ambassadors. }\end{array}$ & $\begin{array}{l}\text { Statistically significant differences } \\
\text { from pre- to posttest in: creating } \\
\text { content that will engage audiences; } \\
\text { establishing credibility with the } \\
\text { audiences; knowing what details to } \\
\text { include and what to leave out; } \\
\text { selecting an appropriate slide design; } \\
\text { delivering content in front of a large } \\
\text { group; holding audiences' attention } \\
\text { for } 15-20 \text { minutes. }\end{array}$ \\
\hline $\begin{array}{l}16] \\
\text { Greene, Zhan, } \\
\text { Anthony, Post \& } \\
\text { Parkhurst (2014) }\end{array}$ & $\begin{array}{l}\text { Ohio State } \\
\text { University }\end{array}$ & $\begin{array}{l}\text { Translating } \\
\text { Engineering } \\
\text { Research to K8 } \\
\text { Students } \\
\text { (TEK8) }\end{array}$ & $\begin{array}{l}\text { Undergraduate } \\
\text { engineering students } \\
\text { matched with faculty } \\
\text { members to conduct a } \\
\text { summer project where } \\
\text { they develop activities } \\
\text { for middle school } \\
\text { classrooms; also } \\
\text { contribute to } 6 \text { weeks } \\
\text { of afterschool } \\
\text { programming. }\end{array}$ & $\begin{array}{l}\text { Programming can impact } \\
\text { undergraduates' self- } \\
\text { efficacy for generating } \\
\text { interest in engineering } \\
\text { among middle school } \\
\text { students. }\end{array}$ & $\begin{array}{l}\text { Investigate } \\
\text { changes in self- } \\
\text { efficacy, reciprocal } \\
\text { learning between } \\
\text { undergraduates } \\
\text { and teachers. }\end{array}$ & $\begin{array}{l}\text { Mixed methods } \\
\text { evaluation study: } \\
\text { interviews, } \\
\text { observations, and } \\
\text { a pre- and post- } \\
\text { self-efficacy } \\
\text { survey. Within } \\
\text { and cross-case } \\
\text { analysis and } \\
\text { discourse } \\
\text { analysis. }\end{array}$ & $\begin{array}{l}\text { Statistically significant gain in self- } \\
\text { efficacy. Undergraduates were already } \\
\text { confident in content knowledge but } \\
\text { gained in self-efficacy for } \\
\text { presentation skills and leading design } \\
\text { challenges. }\end{array}$ \\
\hline $\begin{array}{l}{[21]} \\
\text { DeWaters, } \\
\text { Powers \& } \\
\text { Graham (2006) }\end{array}$ & $\begin{array}{l}\text { Clarkson } \\
\text { University }\end{array}$ & $\begin{array}{l}\text { Partners In } \\
\text { Engineering } \\
\text { (PIE) }\end{array}$ & $\begin{array}{l}\text { Teams of female } \\
\text { engineering students } \\
\text { work with teacher to } \\
\text { provide a } 3 \text { week long } \\
\text { engineering problem } \\
\text { solving class to } 8^{\text {th }} \\
\text { grade students. }\end{array}$ & $\begin{array}{l}\text { Importance of provision of } \\
\text { leadership opportunities } \\
\text { for female undergraduates. }\end{array}$ & None articulated. & $\begin{array}{l}\text { Pre and post- } \\
\text { program survey, } \\
\text { focus group and } \\
\text { problem solving } \\
\text { quiz. }\end{array}$ & $\begin{array}{l}\text { Undergraduates with weak initial } \\
\text { problem solving skills reported } \\
\text { perceived benefits from problem } \\
\text { solving aspect of program. Those with } \\
\text { strong problem solving skills reported } \\
\text { strong satisfaction. Perceived } \\
\text { improvement in public speaking and } \\
\text { teamwork and a rewarding } \\
\text { experience. }\end{array}$ \\
\hline $\begin{array}{l}{[22]} \\
\text { Carberry, } \\
\text { Portsmore \& } \\
\text { Rogers (2007) }\end{array}$ & $\begin{array}{l}\text { Tufts } \\
\text { University }\end{array}$ & $\begin{array}{l}\text { STOMP: } \\
\text { Student } \\
\text { Teacher } \\
\text { Outreach } \\
\text { Mentorship } \\
\text { Program }\end{array}$ & $\begin{array}{l}\text { Undergraduates work } \\
\text { with teachers to } \\
\text { conduct outreach. }\end{array}$ & $\begin{array}{l}\text { Outreach can improve } \\
\text { undergraduates' civic } \\
\text { responsibility, teamwork } \\
\text { and communication skills, } \\
\text { assist in the identification } \\
\text { of career paths, and } \\
\text { prepare them for the } \\
\text { demands of their future } \\
\text { workplaces. }\end{array}$ & $\begin{array}{l}\text { Impact on various } \\
\text { stakeholders. }\end{array}$ & $\begin{array}{l}\text { Experimental } \\
\text { design: } 12 \\
\text { outreach } \\
\text { undergraduates, } \\
7 \text { in introductory } \\
\text { engineering } \\
\text { course. Pre- and } \\
\text { post-test of } \\
\text { knowledge. }\end{array}$ & $\begin{array}{l}\text { No statistically significant differences. } \\
\text { Authors report experimental group } \\
\text { gained in integration of engineering } \\
\text { knowledge into K-12 content and } \\
\text { comparison group gained in being } \\
\text { able to determine relevant features for } \\
\text { a design project. }\end{array}$ \\
\hline
\end{tabular}


Table 1 a (continued). Critical Features of Studies Examining Impact Pre-and Post-Outreach

\begin{tabular}{|c|c|c|c|c|c|c|c|}
\hline Authors & Institution & Program & $\begin{array}{l}\text { Outreach } \\
\text { Model }\end{array}$ & $\begin{array}{l}\text { Premise or Theory } \\
\text { of Action }\end{array}$ & $\begin{array}{l}\text { Purpose or } \\
\text { Research } \\
\text { Questions }\end{array}$ & Methods & $\begin{array}{l}\text { Impact on } \\
\text { Undergraduates } \\
\text { Conducting Outreach }\end{array}$ \\
\hline $\begin{array}{l}{[23]} \\
\text { Jordan-Bloch \& } \\
\text { Cohen (2018) }\end{array}$ & $\begin{array}{l}\text { Stanford } \\
\text { University }\end{array}$ & $\begin{array}{l}\text { Seeds of } \\
\text { Change }\end{array}$ & $\begin{array}{l}\text { Train the trainer } \\
\text { model. Leader } \\
\text { training meetings } \\
\text { where female } \\
\text { undergraduates learn } \\
\text { curriculum and how } \\
\text { to teach/lead others. }\end{array}$ & $\begin{array}{l}\text { Curriculum has a theory of } \\
\text { action including mindsets, } \\
\text { persistence, mentoring and } \\
\text { leadership. }\end{array}$ & $\begin{array}{l}\text { Investigate lessons } \\
\text { learned from the } \\
\text { program. }\end{array}$ & $\begin{array}{l}\text { Reflections from } \\
\text { leader; pre and } \\
\text { post interviews } \\
\text { and surveys for } \\
\text { participants. }\end{array}$ & $\begin{array}{l}\text { Self-reported increase in content } \\
\text { knowledge. } \\
\text { Undergraduates' attention was drawn } \\
\text { to leadership as an area of growth. }\end{array}$ \\
\hline $\begin{array}{l}\text { [24] } \\
\text { Atwood, Patten } \\
\text { \& Pruitt (2010) }\end{array}$ & $\begin{array}{l}\text { University of } \\
\text { California, } \\
\text { Berkeley }\end{array}$ & $\begin{array}{l}\text { Structural } \\
\text { aspects of } \\
\text { biomaterials } \\
\text { (course) } \\
\text { Body By } \\
\text { Design } \\
\text { Outreach } \\
\text { Activity }\end{array}$ & $\begin{array}{l}\text { Undergraduates } \\
\text { develop an outreach } \\
\text { activity for } \\
\text { elementary students } \\
\text { at a museum. } \\
\text { Undergraduates } \\
\text { examined Bloom's } \\
\text { taxonomy, } \\
\text { communication } \\
\text { skills, and teamwork. }\end{array}$ & $\begin{array}{l}\text { Increasing perceived } \\
\text { relevance of engineering to } \\
\text { society may help retain } \\
\text { female undergraduates. } \\
\text { Female students' } \\
\text { communication skill } \\
\text { strengths may be } \\
\text { developed and used } \\
\text { through outreach. }\end{array}$ & $\begin{array}{l}\text { Investigate impact } \\
\text { of incorporating } \\
\text { design and outreach } \\
\text { into undergraduate } \\
\text { course. }\end{array}$ & $\begin{array}{l}\text { Pre- and post- } \\
\text { course survey } \\
\text { administered to } \\
48 \text { undergraduate } \\
\text { students. } \\
\text { Analyses } \\
\text { separated impact } \\
\text { by gender. }\end{array}$ & $\begin{array}{l}\text { Women ranked outreach activity as } \\
\text { being more useful than men. Women } \\
\text { indicated higher self-confidence than } \\
\text { men at the beginning and end of the } \\
\text { course. Men had higher self- } \\
\text { confidence in evaluating written and } \\
\text { analytical work of themselves and } \\
\text { others. Both gained in overall } \\
\text { confidence from pre- to post survey. }\end{array}$ \\
\hline $\begin{array}{l}{[14]} \\
\text { Bigelow (2010) }\end{array}$ & $\begin{array}{l}\text { University of } \\
\text { Dayton }\end{array}$ & $\begin{array}{l}\begin{array}{l}\text { Biomedical } \\
\text { engineering } \\
\text { (course) }\end{array} \\
\text { Prosthetic } \\
\text { Hand } \\
\text { Outreach } \\
\text { Activity }\end{array}$ & $\begin{array}{l}\text { Service learning } \\
\text { activity within } \\
\text { course. } \\
\text { Undergraduates teach } \\
\text { K-12 students about } \\
\text { biomedical research. } \\
\text { Students choose how } \\
\text { they want to conduct } \\
\text { the outreach. }\end{array}$ & $\begin{array}{l}\text { Outreach motivation can } \\
\text { be investigated through } \\
\text { constructs of Valence, } \\
\text { Instrumentality, and } \\
\text { Expectancy. }\end{array}$ & $\begin{array}{l}\text { Investigate impact } \\
\text { of conducting } \\
\text { outreach. }\end{array}$ & $\begin{array}{l}\text { Pre and post- } \\
\text { semester survey } \\
\text { about biomedical } \\
\text { engineering } \\
\text { outreach. Post- } \\
\text { outreach } \\
\text { reflections. }\end{array}$ & $\begin{array}{l}\text { Self-reported changes in their } \\
\text { perceptions of the need for } \\
\text { engineering outreach. } \\
\text { Lessons learned in four areas: setting } \\
\text { up and conducting an outreach } \\
\text { activity; learning to work with young } \\
\text { adults; awareness of engineering; } \\
\text { importance, and role of diversity. }\end{array}$ \\
\hline $\begin{array}{l}{[25]} \\
\text { Sullivan \& } \\
\text { Zarske (2005) }\end{array}$ & $\begin{array}{l}\text { University of } \\
\text { Colorado, } \\
\text { Boulder }\end{array}$ & $\begin{array}{l}\text { K-12 } \\
\text { Engineering } \\
\text { Outreach } \\
\text { Corps }\end{array}$ & $\begin{array}{l}\text { Engineering used as a } \\
\text { vehicle for teaching } \\
\text { science and math. } \\
\text { Undergraduates take } \\
\text { a class to prepare } \\
\text { them to lead } \\
\text { outreach. }\end{array}$ & $\begin{array}{l}\text { Undergraduates gain } \\
\text { knowledge not included in } \\
\text { coursework. Retention of } \\
\text { women and students of } \\
\text { color can improve using an } \\
\text { authentic curriculum that } \\
\text { includes outreach. }\end{array}$ & None articulated. & $\begin{array}{l}\text { Pre- and post- } \\
\text { semester surveys, } \\
\text { observations, } \\
\text { focus group. }\end{array}$ & $\begin{array}{l}\text { Self-reported increases in integrating } \\
\text { engineering fields and integrating } \\
\text { curriculum into K-12 classrooms, } \\
\text { develop K-12 engineering curriculum, } \\
\text { and work with children. Decreases in } \\
\text { confidence to work with K-12 } \\
\text { principals and work with special } \\
\text { needs children. }\end{array}$ \\
\hline
\end{tabular}


Table 1a (continued). Critical Features of Studies Examining Impact Pre-and Post-Outreach

\begin{tabular}{|c|c|c|c|c|c|c|c|}
\hline Authors & Institution & Program & $\begin{array}{l}\text { Outreach } \\
\text { Model }\end{array}$ & $\begin{array}{l}\text { Premise or Theory } \\
\text { of Action }\end{array}$ & $\begin{array}{l}\text { Purpose or } \\
\text { Research } \\
\text { Questions } \\
\end{array}$ & Methods & $\begin{array}{l}\text { Impact on } \\
\text { Undergraduates } \\
\text { Conducting Outreach }\end{array}$ \\
\hline $\begin{array}{l}{[12]} \\
\text { Switzer \& } \\
\text { Benson (2007) }\end{array}$ & $\begin{array}{l}\text { Clemson } \\
\text { University }\end{array}$ & $\begin{array}{l}\text { Engineering } \\
\text { Fundamentals } \\
\text { (Class) } 3 \text { week } \\
\text { design project }\end{array}$ & $\begin{array}{l}\text { Undergraduates } \\
\text { create active learning } \\
\text { tools for middle and } \\
\text { high school students. }\end{array}$ & $\begin{array}{l}\text { Valence Instrumentality } \\
\text { Expectancy theory } \\
\text { (Vroom, 1964). }\end{array}$ & None articulated. & $\begin{array}{l}\text { Survey of } 160 \\
\text { undergraduates } \\
\text { before and after } \\
\text { design project. }\end{array}$ & $\begin{array}{l}\text { Trends but no statistically significant } \\
\text { changes in value of behavior, value of } \\
\text { goal, instrumentality, or expectancy. }\end{array}$ \\
\hline $\begin{array}{l}26] \\
\text { Atwood \& Frey } \\
(2013)\end{array}$ & $\begin{array}{l}\text { Elizabethtown } \\
\text { College }\end{array}$ & $\begin{array}{l}\text { Strength of } \\
\text { materials } \\
\text { (Course) }\end{array}$ & $\begin{array}{l}\text { Outreach performed } \\
\text { as part of } \\
\text { coursework. }\end{array}$ & $\begin{array}{l}\text { Motivation to perform } \\
\text { outreach involves: Valence } \\
\text { (value), Instrumentality } \\
\text { (connection between } \\
\text { activity and progress } \\
\text { towards goals) and } \\
\text { Expectancy (ability and } \\
\text { skills to perform outreach } \\
\text { successfully). } \\
\text { Outreach meets ABET } \\
\text { criteria a, c, f, g, i. }\end{array}$ & $\begin{array}{l}\text { Investigate } \\
\text { gendered impact of } \\
\text { performing } \\
\text { outreach. }\end{array}$ & $\begin{array}{l}\text { Open-ended } \\
\text { reflection after } \\
\text { outreach: What } \\
\text { happened? What } \\
\text { does it mean? What } \\
\text { will you do? Pre- } \\
\text { activity survey on } \\
\text { confidence in } \\
\text { technical } \\
\text { communication. }\end{array}$ & $\begin{array}{l}\text { Value of outreach differed for men } \\
\text { and women: male students } \\
\text { emphasized challenges associated } \\
\text { with outreach and female students } \\
\text { emphasized communication skills and } \\
\text { value of working with children. } \\
\text { Both men and women were aware of } \\
\text { gender inequity in engineering. }\end{array}$ \\
\hline
\end{tabular}


Table 1b. Critical Features of Studies Examining Post-Hoc Impact of Outreach

\begin{tabular}{|c|c|c|c|c|c|c|c|}
\hline Authors & Institution & Program & $\begin{array}{l}\text { Outreach } \\
\text { Model }\end{array}$ & $\begin{array}{l}\text { Premise or } \\
\text { Theory of Action }\end{array}$ & $\begin{array}{l}\text { Purpose or } \\
\text { Research } \\
\text { Questions }\end{array}$ & Methods & $\begin{array}{l}\text { Impact on } \\
\text { Undergraduates } \\
\text { Conducting Outreach }\end{array}$ \\
\hline $\begin{array}{l}\text { [3] } \\
\text { Alley, Haas, } \\
\text { Garner \& Thole } \\
\text { (2015) }\end{array}$ & Various & $\begin{array}{l}\text { Engineering } \\
\text { Ambassadors } \\
\text { Network (EAN) }\end{array}$ & $\begin{array}{l}\text { Three characteristics } \\
\text { of EA program: } 1 . \\
\text { Communication } \\
\text { training through the } \\
\text { workshop; } 2 \text {. } \\
\text { Outreach performed } \\
\text { in middle and high } \\
\text { schools; } 3 \text {. } \\
\text { Ambassadors learn } \\
\text { professional skills } \\
\text { through academic } \\
\text { programs and courses. }\end{array}$ & $\begin{array}{l}\text { Communication training } \\
\text { including online training } \\
\text { will enhance } \\
\text { undergraduate professional } \\
\text { development and increase } \\
\text { Network impact. }\end{array}$ & $\begin{array}{l}\text { Assess impact of } \\
\text { training and } \\
\text { identify critical } \\
\text { features of } \\
\text { individual chapter } \\
\text { success. }\end{array}$ & $\begin{array}{l}\text { Post-workshop } \\
\text { Likert scale } \\
\text { survey of } 170 \\
\text { ambassadors }\end{array}$ & $\begin{array}{l}97 \% \text { of survey respondents responded } \\
\text { that the training exceeded their } \\
\text { expectations; } 75 \% \text { indicated they } \\
\text { could create content that would } \\
\text { engage an outreach audience; } 74 \% \\
\text { create slides that would help the } \\
\text { audience understand information; } \\
72 \% \text { select appropriate slide design; } \\
68 \% \text { provide a helpful critique of a } \\
\text { peer's presentation; } 66 \% \text { know what } \\
\text { to include and what to exclude in an } \\
\text { outreach presentation; } 61 \% \text { integrate } \\
\text { Changing the Conversation messages. }\end{array}$ \\
\hline $\begin{array}{l}{[27]} \\
\text { Haas, Alley, } \\
\text { Garner \& Thole } \\
\text { (2016) }\end{array}$ & Various & $\begin{array}{l}\text { Engineering } \\
\text { Ambassadors } \\
\text { Network (EAN) }\end{array}$ & $\begin{array}{l}\text { Engineering } \\
\text { Ambassadors } \\
\text { Network training } \\
\text { scaled to other new } \\
\text { and existing chapters. }\end{array}$ & $\begin{array}{l}\text { Communication skills are } \\
\text { necessary for } \\
\text { undergraduate engineering } \\
\text { students. }\end{array}$ & None articulated. & $\begin{array}{l}\text { Mixed methods } \\
\text { study: interviews } \\
\text { with } \\
\text { ambassadors and } \\
\text { post-training } \\
\text { survey of } 170 \\
\text { ambassadors. }\end{array}$ & $\begin{array}{l}\text { Undergraduate ambassadors } \\
\text { expressed a personal commitment to } \\
\text { maintaining involvement in outreach } \\
\text { at their institution. Perceived increase } \\
\text { in public speaking confidence skills } \\
\text { and ability to create an effective } \\
\text { outreach presentation. } 95 \% \text { of } \\
\text { respondents thought the training was a } \\
\text { good networking opportunity. } 80 \% \\
\text { indicated being able to communicate } \\
\text { messages from Changing the } \\
\text { Conversation. }\end{array}$ \\
\hline $\begin{array}{l}{[28]} \\
\text { Shabhazi, } \\
\text { Lehnes, Jacobs \& } \\
\text { Mancuso (2016) }\end{array}$ & $\begin{array}{l}\text { Manhattan } \\
\text { College }\end{array}$ & $\begin{array}{l}\text { Engineering } \\
\text { Ambassadors }\end{array}$ & $\begin{array}{l}\text { Variation on EAN; } \\
\text { collaboration between } \\
\text { School of Education } \\
\text { and Health and local } \\
\text { high school. }\end{array}$ & $\begin{array}{l}\text { Outreach can encourage } \\
\text { enrollment in } \\
\text { undergraduate engineering } \\
\text { education minor; improve } \\
\text { engineering students' } \\
\text { presentation skills. }\end{array}$ & None articulated. & $\begin{array}{l}\text { Survey of } 89 \\
\text { undergraduates. }\end{array}$ & $\begin{array}{l}\text { Undergraduates could describe the } \\
\text { lesson they had developed and could } \\
\text { rate peer presentations; reported an } \\
\text { improved understanding of learning } \\
\text { styles. }\end{array}$ \\
\hline
\end{tabular}


Table $1 b$ (continued). Critical Features of Studies Examining Post-Hoc Impact of Outreach

\begin{tabular}{|c|c|c|c|c|c|c|c|}
\hline Authors & Institution & Program & $\begin{array}{l}\text { Outreach } \\
\text { Model }\end{array}$ & $\begin{array}{l}\text { Premise or } \\
\text { Theory of Action }\end{array}$ & $\begin{array}{l}\text { Purpose or } \\
\text { Research } \\
\text { Questions }\end{array}$ & Methods & $\begin{array}{l}\text { Impact on } \\
\text { Undergraduates } \\
\text { Conducting Outreach }\end{array}$ \\
\hline $\begin{array}{l}{[15]} \\
\text { Anagnos, } \\
\text { Lyman-Holt, } \\
\text { Marin-Artieda \& } \\
\text { Momsen (2014) }\end{array}$ & $\begin{array}{l}\text { Oregon State } \\
\text { University } \\
\text { Howard } \\
\text { University }\end{array}$ & $\begin{array}{l}\text { Engineering } \\
\text { Ambassadors }\end{array}$ & $\begin{array}{l}\text { Outreach to campus } \\
\text { visitors, lead tours, } \\
\text { meet with } \\
\text { engineering } \\
\text { professionals who } \\
\text { visit campus. } \\
\text { Training for } \\
\text { ambassadors includes } \\
2 \text { day workshop, and } \\
\text { running a } 4 \text { day } \\
\text { student orientation. }\end{array}$ & $\begin{array}{l}\text { Development of } \\
\text { professional skills is a } \\
\text { priority; STEM and } \\
\text { engineering self-efficacy } \\
\text { can be developed through } \\
\text { outreach; self-efficacy } \\
\text { promotes persistence in } \\
\text { STEM. }\end{array}$ & $\begin{array}{l}\text { Investigate impact } \\
\text { of engineering } \\
\text { ambassador } \\
\text { program on } \\
\text { undergraduate } \\
\text { student } \\
\text { development. }\end{array}$ & $\begin{array}{l}\text { A previously } \\
\text { validated Likert } \\
\text { scale survey was } \\
\text { administered to } \\
51 \text { current and } \\
\text { former } \\
\text { ambassadors at } \\
\text { two programs. }\end{array}$ & $\begin{array}{l}\text { Ambassadors had multiple goals for } \\
\text { participation, including making a } \\
\text { difference in their community, } \\
\text { building a connection with their } \\
\text { engineering program, building their } \\
\text { resume, and having fun. } 85 \% \text { of } \\
\text { ambassadors perceived gains in } \\
\text { confidence to speak in front of others; } \\
80 \% \text { indicated improved confidence } \\
\text { to succeed in engineering; } 100 \% \\
\text { perceived positive effect on their } \\
\text { leadership skills. }\end{array}$ \\
\hline $\begin{array}{l}{[29]} \\
\text { Talbot (2013) }\end{array}$ & $\begin{array}{l}\text { Pennsylvania } \\
\text { State } \\
\text { University }\end{array}$ & $\begin{array}{l}\text { Engineering } \\
\text { Ambassadors } \\
\text { Network (EAN) }\end{array}$ & $\begin{array}{l}\text { Credit bearing } \\
\text { communication } \\
\text { training; focus on } \\
\text { Changing the } \\
\text { Conversation } \\
\text { messages; teams } \\
\text { perform outreach } \\
\text { presentations and } \\
\text { hands-on activities; } \\
\text { leadership training } \\
\text { through required } \\
\text { reading and } \\
\text { meetings. }\end{array}$ & $\begin{array}{l}\text { Employers desire } \\
\text { engineering graduates with } \\
\text { strong communication } \\
\text { skills and outreach can } \\
\text { function as a context for } \\
\text { the professional } \\
\text { development of these } \\
\text { skills. }\end{array}$ & None articulated. & $\begin{array}{l}\text { Surveys of } \\
\text { current } \\
\text { ambassadors on } \\
\text { leadership and } \\
\text { communication } \\
\text { training; post- } \\
\text { training survey } \\
\text { of } 45 \\
\text { ambassadors. }\end{array}$ & $\begin{array}{l}94 \% \text { of respondents developed } \\
\text { increased confidence in presenting } \\
\text { engineering content; } 83 \% \text { reported } \\
\text { being more motivated to learn course } \\
\text { content; } 72 \% \text { agreed outreach } \\
\text { presentation development allowed } \\
\text { them to transfer information outside } \\
\text { of their courses. Post-training surveys } \\
\text { revealed gains in confidence to } \\
\text { engage audiences, establish } \\
\text { credibility with audiences, know what } \\
\text { to include and exclude, how to select } \\
\text { an appropriate slide design, and } \\
\text { deliver content in front of a large } \\
\text { group, and hold audience attention. }\end{array}$ \\
\hline $\begin{array}{l}{[30]} \\
\text { Caldwell, } \\
\text { McCoy, Albers, } \\
\text { Smith \& Parry } \\
\text { (2007) }\end{array}$ & $\begin{array}{l}\text { North Carolina } \\
\text { State } \\
\text { University }\end{array}$ & $\begin{array}{l}\text { RAMP: } \\
\text { Recognizing } \\
\text { Accelerate Math } \\
\text { Potential in } \\
\text { Underrepresented } \\
\text { People }\end{array}$ & $\begin{array}{l}\text { Undergraduate } \\
\text { fellows work } 10 \text { to } \\
15 \text { hours per week. } \\
\text { Weekly meetings, bi- } \\
\text { monthly dinners, } \\
\text { seminars. }\end{array}$ & $\begin{array}{l}\text { Participation may impact } \\
\text { undergraduate and } \\
\text { graduate fellows' career } \\
\text { plans. }\end{array}$ & $\begin{array}{l}\text { Impact on } \\
\text { undergraduate } \\
\text { fellows' } \\
\text { communication } \\
\text { skills, teaching } \\
\text { skills, STEM } \\
\text { content } \\
\text { knowledge. }\end{array}$ & $\begin{array}{l}\text { Survey of } 42 \\
\text { current and } 8 \\
\text { former fellows. }\end{array}$ & $\begin{array}{l}\text { All respondents indicated } \\
\text { participation was a rewarding } \\
\text { experience; } 86 \% \text { recommended } \\
\text { participation. Perceived increases in } \\
\text { communication skills, career } \\
\text { preparation, content knowledge. }\end{array}$ \\
\hline
\end{tabular}


Table $1 b$ (continued). Critical Features of Studies Examining Post-Hoc Impact of Outreach

\begin{tabular}{|c|c|c|c|c|c|c|c|}
\hline Authors & Institution & Program & $\begin{array}{l}\text { Outreach } \\
\text { Model }\end{array}$ & $\begin{array}{l}\text { Premise or } \\
\text { Theory of Action }\end{array}$ & $\begin{array}{l}\text { Purpose or } \\
\text { Research } \\
\text { Questions }\end{array}$ & Methods & $\begin{array}{l}\text { Impact on } \\
\text { Undergraduates } \\
\text { Conducting Outreach }\end{array}$ \\
\hline $\begin{array}{l}\text { [31] } \\
\text { Bates, Krapcho } \\
\text { \& Orantes } \\
(2014)\end{array}$ & $\begin{array}{l}\text { University of } \\
\text { Utah }\end{array}$ & $\begin{array}{l}\text { Ambassador } \\
\text { Program }\end{array}$ & $\begin{array}{l}\text { Undergraduates are } \\
\text { trained to interact } \\
\text { with students of } \\
\text { varying ability, } \\
\text { develop outreach } \\
\text { activities and } \\
\text { presentations; } \\
\text { students participate } \\
\text { in community and } \\
\text { campus events, } \\
\text { including visits to } \\
\text { high school classes. }\end{array}$ & $\begin{array}{l}\text { Undergraduate student } \\
\text { retention can be improved } \\
\text { through community } \\
\text { building. Communities of } \\
\text { ambassadors can promote } \\
\text { practical competence, } \\
\text { personal and social } \\
\text { development, and deeper } \\
\text { engagement. }\end{array}$ & $\begin{array}{l}\text { Investigate } \\
\text { ambassadors' } \\
\text { experiences. }\end{array}$ & $\begin{array}{l}\text { Survey of } 37 \\
\text { ambassadors: } \\
\text { reasons for } \\
\text { participation, } \\
\text { reasons for } \\
\text { enrolling in } \\
\text { engineering, } \\
\text { post-graduation } \\
\text { plans. }\end{array}$ & $\begin{array}{l}\text { Ambassadors reported a much } \\
\text { reduced consideration of switching } \\
\text { major; improved presentation skills; } \\
\text { improved social connections; } \\
\text { improved sense of accomplishment. }\end{array}$ \\
\hline $\begin{array}{l}\text { [7] Yowell } \\
\text { Zarske, Knight } \\
\text { \& Sullivan } \\
\text { (2013) }\end{array}$ & $\begin{array}{l}\text { University of } \\
\text { Colorado, } \\
\text { Boulder }\end{array}$ & $\begin{array}{l}\text { TEAMS: } \\
\text { Tomorrow's } \\
\text { Engineers...creAte, } \\
\text { Imagine, Succeed }\end{array}$ & $\begin{array}{l}\text { Weekly TEAMS } \\
\text { clubs led by } \\
\text { undergraduate } \\
\text { students in } \\
\text { elementary schools. } \\
\text { Boxed activities are } \\
\text { performed. }\end{array}$ & $\begin{array}{l}\text { Value of extracurricular } \\
\text { volunteer activities on } \\
\text { undergraduates includes } \\
\text { improved communication, } \\
\text { content knowledge, and } \\
\text { teaching skills. Impact } \\
\text { particularly for women. }\end{array}$ & $\begin{array}{l}\text { Investigate } \\
\text { leaders' } \\
\text { experiences. }\end{array}$ & $\begin{array}{l}\text { Survey of a total } \\
\text { of } 34 \\
\text { undergraduates } \\
\text { (gathered over a } \\
3 \text { year period). }\end{array}$ & $\begin{array}{l}\text { Respondents indicated impact on oral } \\
\text { communication skills and satisfaction } \\
\text { with engineering studies. Lower } \\
\text { levels of impact on fundamental } \\
\text { engineering skills and future career } \\
\text { plans. Realization of challenge of } \\
\text { communicating to non-technical } \\
\text { audiences. Outreach as a possibility } \\
\text { for action in future jobs. }\end{array}$ \\
\hline $\begin{array}{l}{[32]} \\
\text { Haas, } \\
\text { McElholm, } \\
\text { Renfro, } \\
\text { Herkenham, } \\
\text { Marshall \& } \\
\text { Alley (2013) }\end{array}$ & $\begin{array}{l}\text { Worcester } \\
\text { Polytechnic } \\
\text { University }\end{array}$ & $\begin{array}{l}\text { Engineering } \\
\text { Ambassadors } \\
\text { Network (EAN) }\end{array}$ & $\begin{array}{l}\text { Implementation of } \\
\text { Changing the } \\
\text { Conversation } \\
\text { messages; } \\
\text { performing outreach } \\
\text { to middle and high } \\
\text { school students; } \\
\text { focus on ambassador } \\
\text { professional } \\
\text { development through } \\
\text { academic programs. }\end{array}$ & $\begin{array}{l}\text { Bringing the outreach } \\
\text { programs of multiple } \\
\text { Colleges of Engineering } \\
\text { together can promote } \\
\text { sharing of resources and } \\
\text { information, encourage } \\
\text { mission development, and } \\
\text { develop community for } \\
\text { ambassadors. }\end{array}$ & None articulated. & $\begin{array}{l}\text { Mixed methods } \\
\text { study: } \\
\text { demographics of } \\
\text { ambassadors; } \\
\text { visit logs; } \\
\text { surveys of } 20 \\
\text { ambassadors } \\
\text { from one } \\
\text { institution. }\end{array}$ & $\begin{array}{l}\text { Survey findings revealed } 94 \% \\
\text { developed confidence presenting } \\
\text { engineering content; } 83 \% \text { perceived } \\
\text { participation helped them develop } \\
\text { self-direction and responsibility; } 60 \% \\
\text { indicated a better understanding of } \\
\text { engineering; } 49 \% \text { indicated an } \\
\text { increased interest in engineering } \\
\text { content. }\end{array}$ \\
\hline
\end{tabular}


Table $1 b$ (continued). Critical Features of Studies Examining Post-Hoc Impact of Outreach

\begin{tabular}{|c|c|c|c|c|c|c|c|}
\hline Authors & Institution & Program & $\begin{array}{l}\text { Outreach } \\
\text { Model }\end{array}$ & $\begin{array}{l}\text { Premise or } \\
\text { Theory of Action }\end{array}$ & $\begin{array}{l}\text { Purpose or } \\
\text { Research } \\
\text { Questions }\end{array}$ & Methods & $\begin{array}{l}\text { Impact on } \\
\text { Undergraduates } \\
\text { Conducting Outreach }\end{array}$ \\
\hline $\begin{array}{l}{[33]} \\
\text { Scherrer (2013) }\end{array}$ & $\begin{array}{l}\text { Southern } \\
\text { Polytechnic } \\
\text { State } \\
\text { University }\end{array}$ & $\begin{array}{l}\text { Outreach funded } \\
\text { under NSF BRIGE } \\
\text { grant }\end{array}$ & $\begin{array}{l}\text { Undergraduates visit } \\
\text { high schools to give } \\
\text { presentations about } \\
\text { how engineers "do } \\
\text { good" in the world. }\end{array}$ & None articulated. & $\begin{array}{l}\text { How participation } \\
\text { affects } \\
\text { undergraduates' } \\
\text { interest in } \\
\text { remaining in } \\
\text { program or career; } \\
\text { likelihood of } \\
\text { graduate school; } \\
\text { interest in K-12 } \\
\text { STEM. }\end{array}$ & $\begin{array}{l}\text { Survey of } 11 \\
\text { undergraduates. }\end{array}$ & $\begin{array}{l}\text { Undergraduates reported personal } \\
\text { benefit of communication skills that } \\
\text { transferred beyond outreach. Personal } \\
\text { satisfaction in helping others. } \\
\text { Meaningful outcome of opportunity } \\
\text { to shape others' futures. Outreach } \\
\text { participation did not impact degree } \\
\text { completion or graduate school plans. }\end{array}$ \\
\hline $\begin{array}{l}{[34]} \\
\text { Ciston, Wordsall } \\
\text { \& Swenson } \\
(2010)\end{array}$ & $\begin{array}{l}\text { Northwestern } \\
\text { University }\end{array}$ & $\begin{array}{l}\text { STEP: Summer } \\
\text { Technology \& } \\
\text { Engineering } \\
\text { Program }\end{array}$ & $\begin{array}{l}\text { Undergraduates } \\
\text { conduct } 3 \text { full days of } \\
\text { outreach to } 7^{\text {th }} \text { and } 8^{\text {th }} \\
\text { grade girls. Outreach } \\
\text { includes classroom } \\
\text { activities, field trip, } \\
\text { design project and } \\
\text { research lab tour. }\end{array}$ & $\begin{array}{l}\text { Undergraduate female } \\
\text { student motivation to } \\
\text { participate. }\end{array}$ & None articulated. & $\begin{array}{l}\text { Survey of } 10 \\
\text { undergraduate } \\
\text { and graduate } \\
\text { facilitators who } \\
\text { rated various } \\
\text { sources of } \\
\text { motivation. }\end{array}$ & $\begin{array}{l}\text { Undergraduates felt it was important } \\
\text { for junior high school girls to see } \\
\text { women in engineering and to increase } \\
\text { women's participation in engineering } \\
\text { Money and professor encouragement } \\
\text { were the lowest rated source of } \\
\text { participants' motivation. }\end{array}$ \\
\hline $\begin{array}{l}{[35]} \\
\text { Salzman \& } \\
\text { Strobel (2011) }\end{array}$ & $\begin{array}{l}\text { Purdue } \\
\text { University }\end{array}$ & $\begin{array}{l}\text { Purdue FIRST } \\
\text { Programs (PFP) }\end{array}$ & $\begin{array}{l}\text { FIRST Robotics } \\
\text { competition where } 12 \\
\text { university students } \\
\text { mentor } 18 \text { high } \\
\text { school students. }\end{array}$ & $\begin{array}{l}\text { Service learning and } \\
\text { mentoring bring cognitive } \\
\text { and social benefits to the } \\
\text { undergraduates. }\end{array}$ & $\begin{array}{l}\text { Investigate } \\
\text { motivation of } \\
\text { undergraduates to } \\
\text { mentor high } \\
\text { school students } \\
\text { and what mentors } \\
\text { gain from being } \\
\text { mentors. }\end{array}$ & $\begin{array}{l}\text { Survey of } 10 \\
\text { students with } 1-6 \\
\text { years of } \\
\text { involvement in } \\
\text { program. Open } \\
\text { and deductive } \\
\text { coding. }\end{array}$ & $\begin{array}{l}\text { Motivation to participate influenced } \\
\text { by prior FIRST Robotics } \\
\text { participation. Undergraduates } \\
\text { perceived gains in teaching, } \\
\text { technical, communication, time } \\
\text { management, and leadership skills. } \\
\text { Undergraduates perceived } \\
\text { development of technical and process } \\
\text { skills. }\end{array}$ \\
\hline $\begin{array}{l}{[36]} \\
\text { Bowling, Doyle, } \\
\text { Taylor \& Antes, } \\
(2015)\end{array}$ & $\begin{array}{l}\text { Northern } \\
\text { Kentucky } \\
\text { University }\end{array}$ & $\begin{array}{l}\text { STEM } \\
\text { Ambassadors. }\end{array}$ & $\begin{array}{l}\text { Undergraduates act } \\
\text { as peer mentors, } \\
\text { conduct outreach and } \\
\text { attend recruitment } \\
\text { events. Training } \\
\text { includes facilitating } \\
\text { an orientation } \\
\text { workshop, } \\
\text { assignments, } \\
\text { mentorship, } \\
\text { leadership, and } \\
\text { coaching training. }\end{array}$ & $\begin{array}{l}\text { Academic and social } \\
\text { integration and perceived } \\
\text { belonging to a disciplinary } \\
\text { community promotes } \\
\text { retention of undergraduate } \\
\text { students. }\end{array}$ & $\begin{array}{l}\text { Investigate } \\
\text { retention effects } \\
\text { of } \\
\text { ambassadorship. }\end{array}$ & $\begin{array}{l}\text { Open ended } \\
\text { question survey } \\
\text { of } 10 \\
\text { ambassadors; } \\
\text { statements from } \\
\text { mentees; } \\
\text { retention data. }\end{array}$ & $\begin{array}{l}\text { STEM ambassadors reported learning } \\
\text { lessons of trusting team members, } \\
\text { delegating, being aware of others' } \\
\text { perspectives, realizing it is acceptable } \\
\text { to be vulnerable, maintaining a focus } \\
\text { on 'big picture' issues, and } \\
\text { recognizing and honoring } \\
\text { commitments. } \\
\text { Retention of STEM students who } \\
\text { interacted with ambassadors was } \\
\text { higher than those who had not. }\end{array}$ \\
\hline
\end{tabular}


Table $1 b$ (continued). Critical Features of Studies Examining Post-Hoc Impact of Outreach

\begin{tabular}{|c|c|c|c|c|c|c|c|}
\hline Authors & Institution & Program & $\begin{array}{l}\text { Outreach } \\
\text { Model }\end{array}$ & $\begin{array}{l}\text { Premise or } \\
\text { Theory of Action }\end{array}$ & $\begin{array}{l}\text { Purpose or } \\
\text { Research } \\
\text { Questions }\end{array}$ & Methods & $\begin{array}{l}\text { Impact on } \\
\text { Undergraduates } \\
\text { Conducting Outreach }\end{array}$ \\
\hline $\begin{array}{l}\text { [37] } \\
\text { Epstein, Mire, } \\
\text { Ramsey, Gareis, } \\
\text { Davidson, Jones, } \\
\text { Slosberg \& Bras } \\
\text { (2010) }\end{array}$ & $\begin{array}{l}\text { Massachusetts } \\
\text { Institute of } \\
\text { Technology }\end{array}$ & $\begin{array}{l}\text { Terrascope Youth } \\
\text { Radio (TYR) }\end{array}$ & $\begin{array}{l}\text { Engineering and } \\
\text { science } \\
\text { undergraduates act as } \\
\text { mentors for a } 6 \text { week } \\
\text { summer program } \\
\text { where they with } \\
\text { teenagers to produce } \\
\text { radio and audio } \\
\text { programming on } \\
\text { environmental topics. }\end{array}$ & $\begin{array}{l}\text { Generational links between } \\
\text { older and younger } \\
\text { students. }\end{array}$ & $\begin{array}{l}\text { Investigate } \\
\text { successes, } \\
\text { challenges, } \\
\text { rewards of being a } \\
\text { mentor. }\end{array}$ & $\begin{array}{l}\text { Survey of open- } \\
\text { ended questions } \\
\text { responded to by } \\
4 \text { out of } 8 \\
\text { mentors. }\end{array}$ & $\begin{array}{l}\text { Undergraduates reported learning } \\
\text { specific mentoring skills including } \\
\text { giving guidance and engaging in } \\
\text { critical thinking. One respondent } \\
\text { indicated improving these skills } \\
\text { herself. }\end{array}$ \\
\hline $\begin{array}{l}\text { [19] Garner, } \\
\text { Alley, Haas, } \\
\text { Sontgerath \& } \\
\text { Kaplan (2017) }\end{array}$ & Various & $\begin{array}{l}\text { Engineering } \\
\text { Ambassadors } \\
\text { Network (EAN) }\end{array}$ & $\begin{array}{l}\text { Outreach to middle } \\
\text { and high school } \\
\text { students; } \\
\text { communication } \\
\text { training for } \\
\text { ambassadors. }\end{array}$ & $\begin{array}{l}\text { Outreach can be a form of } \\
\text { professional development } \\
\text { and can impact the } \\
\text { ambassadors' self- } \\
\text { perceptions, purpose and } \\
\text { goals, beliefs, and } \\
\text { possibilities for action. }\end{array}$ & $\begin{array}{l}\text { Investigate } \\
\text { purpose of the } \\
\text { hands-on activity } \\
\text { for students in an } \\
\text { ambassador role; } \\
\text { process of } \\
\text { developing hands- } \\
\text { on activity. }\end{array}$ & $\begin{array}{l}\text { Case study with } \\
\text { embedded units. } \\
\text { Interviews with } \\
8 \text { ambassadors } \\
\text { after a week- } \\
\text { long training. }\end{array}$ & $\begin{array}{l}\text { Undergraduates perceive that hands- } \\
\text { on activities serve a learning purpose } \\
\text { and engage students in what it means } \\
\text { to be an engineer. Ambassadors had } \\
\text { to consider the integration of content, } \\
\text { pedagogy, and technical constraints. }\end{array}$ \\
\hline $\begin{array}{l}38] \\
\text { Garner, Alley, } \\
\text { Haas \& Kaplan } \\
(2016)\end{array}$ & Various & $\begin{array}{l}\text { Engineering } \\
\text { Ambassadors } \\
\text { Network (EAN) }\end{array}$ & $\begin{array}{l}\text { Three characteristics } \\
\text { of EA program: } 1 . \\
\text { Communication } \\
\text { training through the } \\
\text { workshop; } 2 \text {. } \\
\text { Outreach performed } \\
\text { in middle and high } \\
\text { schools; } 3 \text {. } \\
\text { Ambassadors learn } \\
\text { professional skills } \\
\text { through academic } \\
\text { programs and } \\
\text { courses. }\end{array}$ & $\begin{array}{l}\text { Professional identity } \\
\text { development occurs as a } \\
\text { by-product of participating } \\
\text { as an engineering } \\
\text { ambassador and } \\
\text { performing outreach; } \\
\text { particular benefit if the } \\
\text { ambassador is from an } \\
\text { underrepresented group. } \\
\text { Use of Dynamic Systems } \\
\text { Model of Role Identity as } \\
\text { a theoretical framework. }\end{array}$ & $\begin{array}{l}\text { How identities } \\
\text { inform motivation } \\
\text { to become an } \\
\text { ambassador; how } \\
\text { training } \\
\text { crystallizes } \\
\text { identity as an } \\
\text { ambassador for } \\
\text { the field of } \\
\text { engineering; } \\
\text { features of the } \\
\text { workshop that } \\
\text { promote identity } \\
\text { formation. }\end{array}$ & $\begin{array}{l}\text { Case study with } \\
\text { embedded units. } \\
\text { Interviews with } \\
\text { purposeful } \\
\text { sample of } 6 \\
\text { undergraduate } \\
\text { ambassadors. }\end{array}$ & $\begin{array}{l}\text { Ambassadors' beliefs echo the } \\
\text { problems and solutions included in } \\
\text { Changing the Conversation; } \\
\text { ambassadors' professional goals are } \\
\text { broadly aligned with Changing the } \\
\text { Conversation; Ambassadors' own } \\
\text { professional development was not a } \\
\text { prominent motivation to participate in } \\
\text { outreach; action possibilities for } \\
\text { outreach were clear as a result of the } \\
\text { workshop training; ambassadors' } \\
\text { choice of outreach presentations } \\
\text { reflected existing interests. }\end{array}$ \\
\hline
\end{tabular}


Table $1 b$ (continued). Critical Features of Studies Examining Post-Hoc Impact of Outreach

\begin{tabular}{|c|c|c|c|c|c|c|c|}
\hline Authors & Institution & Program & $\begin{array}{l}\text { Outreach } \\
\text { Model }\end{array}$ & $\begin{array}{l}\text { Premise or Theory } \\
\text { of Action }\end{array}$ & $\begin{array}{l}\text { Purpose or } \\
\text { Research } \\
\text { Questions } \\
\end{array}$ & Methods & $\begin{array}{l}\text { Impact on } \\
\text { Undergraduates } \\
\text { Conducting Outreach }\end{array}$ \\
\hline $\begin{array}{l}\text { [4] Garner, Haas, } \\
\text { Alley \& Kaplan } \\
\text { (2018) }\end{array}$ & Various & $\begin{array}{l}\text { Engineering } \\
\text { Ambassadors } \\
\text { Network (EAN) }\end{array}$ & $\begin{array}{l}\text { Three characteristics } \\
\text { of EA program: } 1 . \\
\text { Communication } \\
\text { training through the } \\
\text { workshop; } 2 \text {. } \\
\text { Outreach performed } \\
\text { in middle and high } \\
\text { schools; } 3 \text {. } \\
\text { Ambassadors learn } \\
\text { professional skills } \\
\text { through academic } \\
\text { programs and } \\
\text { courses. }\end{array}$ & $\begin{array}{l}\text { Training for participation } \\
\text { in outreach provides a } \\
\text { context for undergraduate } \\
\text { identity development; } \\
\text { theoretical framework of } \\
\text { Dynamic Systems Model } \\
\text { of Role Identity used to } \\
\text { understand identity } \\
\text { development processes and } \\
\text { outcomes. }\end{array}$ & $\begin{array}{l}\text { How do } \\
\text { individuals' } \\
\text { existing identities } \\
\text { motivate them to } \\
\text { become } \\
\text { ambassadors? } \\
\text { How did the } \\
\text { training workshop } \\
\text { shape } \\
\text { undergraduates' } \\
\text { understanding of } \\
\text { the ambassador } \\
\text { role? } \\
\text { Which features of } \\
\text { the workshop } \\
\text { promote identity } \\
\text { development? }\end{array}$ & $\begin{array}{l}\text { Qualitative case } \\
\text { study with } 6 \\
\text { embedded units. } \\
\text { Interviews were } \\
\text { coded using the } \\
\text { DSMRI } \\
\text { theoretical } \\
\text { framework; cases } \\
\text { were } \\
\text { synthesized; } \\
\text { cross-case } \\
\text { analysis. }\end{array}$ & $\begin{array}{l}\text { Incoming role identities of the } \\
\text { ambassadors were aligned with the } \\
\text { messages of Changing the } \\
\text { Conversation and the mission of the } \\
\text { EAN organization; the training } \\
\text { workshop allowed sharing of existing } \\
\text { engineering knowledge and skills and } \\
\text { ways to hone and communicate } \\
\text { knowledge; presentation skills } \\
\text { training and the social context } \\
\text { emerged as important workshop } \\
\text { features. }\end{array}$ \\
\hline $\begin{array}{l}39] \\
\text { McFalls, } \\
\text { Grimes, } \\
\text { Mohammadi- } \\
\text { Aragh, Sullivan } \\
\text { \& Warncok } \\
(2015)\end{array}$ & $\begin{array}{l}\text { Mississippi } \\
\text { State University }\end{array}$ & None provided. & $\begin{array}{l}\text { Undergraduates } \\
\text { participate in } \\
\text { residential summer } \\
\text { program aiming to } \\
\text { recruit students and } \\
\text { inform them about } \\
\text { engineering. } \\
\text { Outreach includes } \\
\text { research, tours. }\end{array}$ & None articulated. & $\begin{array}{l}\text { Investigate how } \\
\text { the experiences } \\
\text { for the } \\
\text { undergraduates } \\
\text { can be improved. }\end{array}$ & $\begin{array}{l}\text { Autoethnographic } \\
\text { study with open } \\
\text { ended prompts } \\
\text { about program } \\
\text { goals and their } \\
\text { experiences } \\
\text { completed by } 2 \\
\text { participants. }\end{array}$ & $\begin{array}{l}\text { Undergraduates had an existing } \\
\text { engineering identity upon beginning } \\
\text { participation. Respondents reported } \\
\text { learning along with students. } \\
\text { Increased appreciation for } \\
\text { interdisciplinary nature of } \\
\text { engineering, increased organizational } \\
\text { and planning skills. }\end{array}$ \\
\hline
\end{tabular}


Table $1 b$ (continued). Critical Features of Studies Examining Post-Hoc Impact of Outreach

\begin{tabular}{|c|c|c|c|c|c|c|c|}
\hline Authors & Institution & Program & $\begin{array}{l}\text { Outreach } \\
\text { Model }\end{array}$ & $\begin{array}{l}\text { Premise or } \\
\text { Theory of Action }\end{array}$ & $\begin{array}{l}\text { Purpose or } \\
\text { Research } \\
\text { Questions }\end{array}$ & Methods & $\begin{array}{l}\text { Impact on } \\
\text { Undergraduates } \\
\text { Conducting Outreach }\end{array}$ \\
\hline $\begin{array}{l}{[40]} \\
\text { Mustafa \& } \\
\text { Freese (2018) }\end{array}$ & $\begin{array}{l}\text { California State } \\
\text { University, } \\
\text { Chico }\end{array}$ & Imagineer Day & $\begin{array}{l}\text { Undergraduates lead } \\
\text { an annual one-day } \\
\text { event with hands-on } \\
\text { lab experience } \\
\text { demonstration of basic } \\
\text { engineering concepts. }\end{array}$ & $\begin{array}{l}\text { Society for Women } \\
\text { Engineer group of female } \\
\text { undergraduates providing } \\
\text { outreach to } \mathrm{K}-8^{\text {th }} \text { grade } \\
\text { girls. }\end{array}$ & $\begin{array}{l}\text { Evaluate the } \\
\text { impact of } \\
\text { participation on } \\
\text { female } \\
\text { undergraduates. }\end{array}$ & $\begin{array}{l}\text { Survey of } \\
\text { participating } \\
\text { undergraduates. }\end{array}$ & $\begin{array}{l}\text { Undergraduates perceived impact on } \\
\text { time management, public speaking, } \\
\text { teamwork, leadership and } \\
\text { communication skills. Respondents } \\
\text { did not report gains in understanding } \\
\text { of engineering but grew interest in } \\
\text { promoting STEM. }\end{array}$ \\
\hline $\begin{array}{l}\text { [41] } \\
\text { Olds, Patel, } \\
\text { Yalvac, Kanter } \\
\text { \& Goel (2004) }\end{array}$ & $\begin{array}{l}\text { Northwestern } \\
\text { University }\end{array}$ & $\begin{array}{l}\text { Project } \\
\text { Prosthesis: } \\
\text { Helping Hands } \\
(\mathrm{PPHH})\end{array}$ & $\begin{array}{l}\text { Students develop a } 600 \\
\text { minute module and } \\
\text { test it in a classroom. }\end{array}$ & $\begin{array}{l}\text { Integration of learning } \\
\text { theory with societal } \\
\text { benefit of engineering. }\end{array}$ & $\begin{array}{l}\text { Investigate gains } \\
\text { in undergraduates' } \\
\text { understanding. }\end{array}$ & $\begin{array}{l}\text { Survey with } \\
\text { open-ended } \\
\text { questions. }\end{array}$ & $\begin{array}{l}\text { All respondents indicated interaction } \\
\text { with faculty and students was one } \\
\text { reason for participation. } 90 \% \\
\text { perceived a gain in understanding } \\
\text { and utilization of engineering design } \\
\text { processes. } 90 \% \text { reported an increase } \\
\text { in interest in teaching and curriculum } \\
\text { development. } 80 \% \text { perceived an } \\
\text { increase in communication skills. }\end{array}$ \\
\hline $\begin{array}{l}{[42]} \\
\text { Olds, Kanter, } \\
\text { Knudson \& } \\
\text { Mehta (2003) }\end{array}$ & $\begin{array}{l}\text { Northwestern } \\
\text { University }\end{array}$ & $\begin{array}{l}\text { Biomedical } \\
\text { Engineering } \\
\text { Outreach }\end{array}$ & $\begin{array}{l}\text { An 8-12 class period } \\
\text { module developed by } \\
\text { undergraduates and } \\
\text { faculty members, } \\
\text { designed for middle } \\
\text { school students. } \\
\text { Undergraduates attend } \\
\text { classes and discuss } \\
\text { homework, guide } \\
\text { activities, manage } \\
\text { structure and time, } \\
\text { observe, take notes. }\end{array}$ & None articulated. & $\begin{array}{l}\text { Investigate change } \\
\text { in undergraduates' } \\
\text { understanding of } \\
\text { teaching and } \\
\text { learning process. }\end{array}$ & $\begin{array}{l}\text { Survey with } \\
\text { open-ended } \\
\text { questions. }\end{array}$ & $\begin{array}{l}\text { Undergraduates reflected on content } \\
\text { and learning outcomes. Summary of } \\
\text { findings indicated gains in } \\
\text { leadership, management and } \\
\text { communication skills. }\end{array}$ \\
\hline
\end{tabular}


Table $1 b$ (continued). Critical Features of Studies Examining Post-Hoc Impact of Outreach

\begin{tabular}{|c|c|c|c|c|c|c|c|}
\hline Authors & Institution & Program & $\begin{array}{l}\text { Outreach } \\
\text { Model }\end{array}$ & $\begin{array}{l}\text { Premise or } \\
\text { Theory of Action }\end{array}$ & $\begin{array}{l}\text { Purpose or } \\
\text { Research } \\
\text { Questions } \\
\end{array}$ & Methods & $\begin{array}{l}\text { Impact on } \\
\text { Undergraduates } \\
\text { Conducting Outreach }\end{array}$ \\
\hline $\begin{array}{l}\text { [43] } \\
\text { Rivas \& Olmsted } \\
\text { (2013) }\end{array}$ & Iridescent & $\begin{array}{l}\text { Be a Scientist! } \\
\text { (BAS) }\end{array}$ & $\begin{array}{l}\text { Undergraduates design } \\
\text { and teach family } \\
\text { science workshops to } \\
\text { groups of } \\
\text { underrepresented } \\
\text { elementary school } \\
\text { children and families } \\
\text { in museums and } \\
\text { schools. }\end{array}$ & $\begin{array}{l}\text { Engineering students can } \\
\text { act as instructors. }\end{array}$ & $\begin{array}{l}\text { Impact of the } \\
\text { project, including } \\
\text { on undergraduates. }\end{array}$ & $\begin{array}{l}\text { Surveys, } \\
\text { interviews, logs, } \\
\text { observations, } \\
\text { and concept } \\
\text { maps. }\end{array}$ & $\begin{array}{l}\text { Undergraduates commented that they } \\
\text { learned practical skills, public } \\
\text { speaking and collaborative skills, and } \\
\text { the ability to simplify and break } \\
\text { down concepts. }\end{array}$ \\
\hline $\begin{array}{l}\text { [44] } \\
\text { Albers, Smith, } \\
\text { Caldwell, } \\
\text { McCoy, } \\
\text { Bottomly, \& } \\
\text { Parry (2008) }\end{array}$ & $\begin{array}{l}\text { North Carolina } \\
\text { State University }\end{array}$ & $\begin{array}{l}\text { RAMP: } \\
\text { Recognizing } \\
\text { Accelerate Math } \\
\text { Potential in } \\
\text { Underrepresente } \\
\text { d People }\end{array}$ & $\begin{array}{l}\text { Undergraduate and } \\
\text { graduate students } \\
\text { conduct hands on } \\
\text { activity based outreach } \\
\text { to math and energy } \\
\text { clubs in local inner } \\
\text { city elementary } \\
\text { schools. }\end{array}$ & $\begin{array}{l}\text { Participation may impact } \\
\text { undergraduate and } \\
\text { graduate fellows' career } \\
\text { plans. }\end{array}$ & $\begin{array}{l}\text { Impact on various } \\
\text { stakeholder } \\
\text { groups. }\end{array}$ & $\begin{array}{l}\text { Surveys of } \\
\text { undergraduate } \\
\text { and graduate } \\
\text { fellows in which } \\
\text { respondents } \\
\text { indicated } \\
\text { agreement with } \\
\text { various } \\
\text { statements. } \\
\end{array}$ & $\begin{array}{l}\text { Fellows indicated positive impacts on } \\
\text { their personal development, } \\
\text { educational relationships, and STEM } \\
\text { knowledge. }\end{array}$ \\
\hline $\begin{array}{l}\text { [17] Ross, } \\
\text { Fletcher, } \\
\text { Theamotharan \& } \\
\text { Garcia (2018) }\end{array}$ & $\begin{array}{l}\text { Florida } \\
\text { International } \\
\text { University }\end{array}$ & $\begin{array}{l}\text { Verizon } \\
\text { Innovative } \\
\text { Learning (VIL) } \\
\text { Program }\end{array}$ & $\begin{array}{l}\text { Undergraduates } \\
\text { hosting middle school } \\
\text { underrepresented male } \\
\text { students at a summer } \\
\text { camp. Engineering } \\
\text { habits of mind, design } \\
\text { theory, computing, 3D } \\
\text { printing. Two days of } \\
\text { training for facilitators. }\end{array}$ & $\begin{array}{l}\text { Social Identity Theory: } \\
\text { performance/competence, } \\
\text { interest, recognition of } \\
\text { competence. Mentor } \\
\text { relationships can be } \\
\text { mutually beneficial, in } \\
\text { part due to similarity } \\
\text { between mentor and } \\
\text { mentee. }\end{array}$ & $\begin{array}{l}\text { Role of race and } \\
\text { gender in student } \\
\text { mentor } \\
\text { relationships; } \\
\text { implications of } \\
\text { mentor } \\
\text { relationships on } \\
\text { STEM identity } \\
\text { development of } \\
\text { the student } \\
\text { mentors. }\end{array}$ & $\begin{array}{l}\text { Survey including } \\
\text { open-ended } \\
\text { questions. } \\
\text { Inductive and } \\
\text { deductive coding } \\
\text { based on Social } \\
\text { Identity theory } \\
\text { Thematic coding } \\
\text { for race, } \\
\text { altruism, and } \\
\text { gender. }\end{array}$ & $\begin{array}{l}\text { Undergraduates entered program } \\
\text { with a pre-existing STEM identity. } \\
\text { Mentoring highlighted altruism and } \\
\text { raised salience of identity, where } \\
\text { identity was defined via } \\
\text { performance/competence and } \\
\text { interest. }\end{array}$ \\
\hline $\begin{array}{l}\text { [45] } \\
\text { Frey \& Atwood } \\
\text { (2013) }\end{array}$ & $\begin{array}{l}\text { Elizabethtown } \\
\text { College }\end{array}$ & $\begin{array}{l}\text { Strength of } \\
\text { Materials (Class) }\end{array}$ & $\begin{array}{l}\text { Service learning in } \\
\text { which undergraduates } \\
\text { create an outreach } \\
\text { activity within } \\
\text { coursework. }\end{array}$ & $\begin{array}{l}\text { Literature derived need } \\
\text { for engineers who can } \\
\text { convey information and } \\
\text { relate to younger } \\
\text { students; need for } \\
\text { diversity. }\end{array}$ & $\begin{array}{l}\text { Investigate impact } \\
\text { of participation on } \\
\text { undergraduates' } \\
\text { recognition of the } \\
\text { need for outreach. }\end{array}$ & $\begin{array}{l}\text { Open-ended } \\
\text { individual } \\
\text { reflection: What } \\
\text { happened? What } \\
\text { does it mean? } \\
\text { What will you } \\
\text { do? }\end{array}$ & $\begin{array}{l}77 \% \text { of respondents were enthusiastic } \\
\text { about continuing outreach after } \\
\text { graduation. Undergraduates felt that } \\
\text { instilling enthusiasm in younger } \\
\text { students was rewarding. }\end{array}$ \\
\hline
\end{tabular}


Table $1 b$ (continued). Critical Features of Studies Examining Post-Hoc Impact of Outreach

\begin{tabular}{|c|c|c|c|c|c|c|c|}
\hline Authors & Institution & Program & $\begin{array}{l}\text { Outreach } \\
\text { Model }\end{array}$ & $\begin{array}{l}\text { Premise or } \\
\text { Theory of Action }\end{array}$ & $\begin{array}{l}\text { Purpose or } \\
\text { Research } \\
\text { Questions } \\
\end{array}$ & Methods & $\begin{array}{l}\text { Impact on } \\
\text { Undergraduates } \\
\text { Conducting Outreach }\end{array}$ \\
\hline $\begin{array}{l}{[46]} \\
\text { Jahan, } \\
\text { Sukumaran, } \\
\text { Head \& Keil } \\
(2012)\end{array}$ & $\begin{array}{l}\text { Rowan } \\
\text { University }\end{array}$ & Project AWE & $\begin{array}{l}\text { Undergraduates and } \\
\text { faculty work together } \\
\text { to provide a } 2 \text { day } \\
\text { camp for middle } \\
\text { school girls that } \\
\text { includes lecture and } \\
\text { lab activities, tours, } \\
\text { and field trips. }\end{array}$ & $\begin{array}{l}\text { Value of mentoring and } \\
\text { potential reciprocal } \\
\text { benefits. }\end{array}$ & None articulated. & $\begin{array}{l}\text { Undergraduates } \\
\text { provided quotes } \\
\text { to supplement } \\
\text { evaluation of } \\
\text { program. }\end{array}$ & $\begin{array}{l}\text { Mentors perceived the experience to } \\
\text { be educational; they had to strike a } \\
\text { balance between being an authority } \\
\text { figure and a friend. Perceived } \\
\text { increase in personal responsibility. }\end{array}$ \\
\hline $\begin{array}{l}{[47]} \\
\text { Brown \& } \\
\text { Thomas (2011) }\end{array}$ & $\begin{array}{l}\text { Virginia } \\
\text { Polytechnic } \\
\text { Institute and } \\
\text { State University }\end{array}$ & $\begin{array}{l}\text { National Society } \\
\text { of Black } \\
\text { Engineers } \\
\text { Technical } \\
\text { Outreach } \\
\text { Community Help } \\
\text { (NSBE TORCH) }\end{array}$ & $\begin{array}{l}\text { Service learning that } \\
\text { includes fundraising } \\
\text { and presentations to K- } \\
12 \text { audiences that visit } \\
\text { campus. }\end{array}$ & $\begin{array}{l}\text { Participation in service } \\
\text { learning and outreach can } \\
\text { impact motivation }\end{array}$ & $\begin{array}{l}\text { Investigate the } \\
\text { impact of } \\
\text { participation on } \\
\text { college students. }\end{array}$ & $\begin{array}{l}\text { Surveys } \\
\text { including } \\
\text { demographic } \\
\text { information, } \\
\text { ranking } \\
\text { statements, and } \\
\text { open-ended } \\
\text { questions of } \\
\text { undergraduate } \\
\text { students. }\end{array}$ & $\begin{array}{l}\text { Thematic statements referred to } \\
\text { students valuing the technical } \\
\text { aspects of their education, the } \\
\text { importance of volunteering, a } \\
\text { commitment to continuing with } \\
\text { outreach throughout their career, and } \\
\text { appreciating applications of } \\
\text { technical skills to other areas of life. }\end{array}$ \\
\hline $\begin{array}{l}{[48]} \\
\text { Greene, Post \& } \\
\text { Abrams (2015) }\end{array}$ & $\begin{array}{l}\text { Ohio State } \\
\text { University }\end{array}$ & $\begin{array}{l}\text { Hometown } \\
\text { Ambassadors } \\
\text { (HA) }\end{array}$ & $\begin{array}{l}\text { Early career engineers } \\
\text { perform outreach to } \\
\text { high school students } \\
\text { without explicitly } \\
\text { recruiting to the } \\
\text { university. }\end{array}$ & $\begin{array}{l}\text { Value of upperclass } \\
\text { undergraduates providing } \\
\text { near peer mentorship and } \\
\text { acting as role models to } \\
\text { high school students. }\end{array}$ & None articulated. & $\begin{array}{l}\text { Survey of } \\
\text { alumni. }\end{array}$ & $\begin{array}{l}\text { Alumni reported changes in } \\
\text { understanding about fields they were } \\
\text { unaware of previously. }\end{array}$ \\
\hline $\begin{array}{l}49] \\
\text { Chakravartula, } \\
\text { Ando, Li, Gupta } \\
\text { \& Pruitt (2006) }\end{array}$ & $\begin{array}{l}\text { University of } \\
\text { California, } \\
\text { Berkeley }\end{array}$ & $\begin{array}{l}\text { Structural } \\
\text { Aspects of } \\
\text { Biomaterials } \\
\text { (Class) }\end{array}$ & $\begin{array}{l}\text { Undergraduates } \\
\text { develop outreach } \\
\text { during class time. } \\
\text { Outreach delivered to } \\
4^{\text {th }} \text { and } 5^{\text {th }} \text { grade } \\
\text { students at field trip to } \\
\text { Lawrence Hall of } \\
\text { Science. }\end{array}$ & $\begin{array}{l}\text { Undergraduates' deep } \\
\text { learning of content results } \\
\text { from teaching it; outreach } \\
\text { teaches undergraduates } \\
\text { about the } \\
\text { interdisciplinary nature of } \\
\text { engineering and improves } \\
\text { teamwork skills. }\end{array}$ & None articulated. & $\begin{array}{l}\text { Observations and } \\
\text { self-evaluations; } \\
\text { written } \\
\text { assignments; } \\
\text { final project } \\
\text { product. }\end{array}$ & $\begin{array}{l}\text { Undergraduates reported learning } \\
\text { about teaching and simplifying } \\
\text { topics; desire to help female students } \\
\text { become interested in biomedical } \\
\text { engineering. }\end{array}$ \\
\hline
\end{tabular}


Table $1 b$ (continued). Critical Features of Studies Examining Post-Hoc Impact of Outreach

\begin{tabular}{|c|c|c|c|c|c|c|c|}
\hline Authors & Institution & Program & $\begin{array}{l}\text { Outreach } \\
\text { Model }\end{array}$ & $\begin{array}{l}\text { Premise or } \\
\text { Theory of Action }\end{array}$ & $\begin{array}{l}\text { Purpose or } \\
\text { Research } \\
\text { Questions }\end{array}$ & Methods & $\begin{array}{l}\text { Impact on } \\
\text { Undergraduates } \\
\text { Conducting Outreach }\end{array}$ \\
\hline $\begin{array}{l}\text { [50] } \\
\text { Pomales-Garcia, } \\
\text { Suarez, Padovani } \\
\text { \& Alvarez } \\
(2018)\end{array}$ & $\begin{array}{l}\text { University of } \\
\text { Puerto Rico }\end{array}$ & $\begin{array}{l}\text { Materials Science } \\
\text { and Engineering } \\
\text { (MSE) Clubs }\end{array}$ & $\begin{array}{l}\text { Year round Material } \\
\text { Science and } \\
\text { Engineering clubs; } \\
\text { summer program for } \\
\text { high school students } \\
\text { and teachers. } \\
\text { Undergraduates } \\
\text { received training on } \\
\text { nanotechnology } \\
\text { demonstrations and } \\
\text { presented them to low } \\
\text { income middle and } \\
\text { high school students. }\end{array}$ & None articulated. & $\begin{array}{l}\text { Program } \\
\text { evaluation results } \\
\text { reported. }\end{array}$ & Participation log. & $\begin{array}{l}\text { Anecdotal quotes referred to } \\
\text { becoming a better mentor, } \\
\text { communicating difficult topics, } \\
\text { giving back to society, and helping } \\
\text { to discover engineering } \\
\text { opportunities. }\end{array}$ \\
\hline $\begin{array}{l}{[51]} \\
\text { Steele \& } \\
\text { Waggenspack } \\
(2018)\end{array}$ & $\begin{array}{l}\text { Louisiana State } \\
\text { University }\end{array}$ & $\begin{array}{l}\text { STEP: STEM } \\
\text { Talent Expansion } \\
\text { Program }\end{array}$ & $\begin{array}{l}\text { Upper level peer } \\
\text { mentor undergraduates } \\
\text { assist with summer } \\
\text { bridge camp. }\end{array}$ & None articulated. & None articulated. & Retention rates. & $\begin{array}{l}\text { Peer mentors were more likely to be } \\
\text { retained than undergraduate students } \\
\text { who had not participated as a mentor. }\end{array}$ \\
\hline
\end{tabular}




\section{References}

1. Amy A. Bergerson, Bryan K. Hotchkiss, and Cynthia Furse, "Outreach and Identity Development: New Perspectives on College Student Persistence," Journal of College Student Retention, vol. 16, no. 2, (2014-2015), pp.165-185.

2. J.P. Bean, "Nine themes of college student retention," In A. Seidman (ed,) College Student Retention: Formula for Student Success (p.215-243). Westport, CT: Praeger.

3. Christine Haas, Michael Alley, Joanna K. Garner, and Karen Thole, "Engineering Ambassadors Network: Progress in 2015 on Creating a National Network of Ambassadors," paper presented at 2016 ASEE Annual Conference and Exposition (New Orleans, Louisiana: ASEE, June 2016).

4. Joanna K. Garner, Michael P. Alley, Christine Haas and Avi Kaplan, "The Emergence of Outreach Ambassador Role Identities in Undergraduate Engineering Students," Journal of STEM Outreach, vol. 1, (2018, October), pp.1-18.

5. Mark Newman and David Gough, "Systematic Reviews in Educational Research: Methodology, Perspectives and Application," In Olaf Zawacki-Richter, Michael Kerres, Svenja Bedenlier, Melissa Bond and Katia Buntins (eds.), Systematic Reviews in Educational Research: Methodology, Perspectives, and Application, Springer, 2019, pp.3-22.

6. Victor H. Vroom, Work and Motivation, Wiley, 1964.

7. Albert Bandura, "Self-efficacy: Towards a Unifying Theory of Behavior Change," Psychological Review vol 84, no. 2, 1977, pp. 191-215.

8. Janet L. Yowell, Malinda S. Zarske, Daniel Knight, and Jacquelyn F. Sullivan, "Impact of TEAMS Clubs: An Afterschool Engineering Enrichment Program that Impacts K12 Students and College Student Leaders," paper presented at 2013 ASEE Annual Conference and Exposition (Atlanta, Georgia: ASEE, June 2013).

9. Alexander W. Astin and Linda J. Sax, "How Undergraduates are Affected by Service Participation," Journal of College Student Development, vol 39, no.3, (1998), pp. 251263.

10. John Turner, Michael Hogg, Penelope J. Oakes, Stephen D. Reicher, and Margaret S. Wetherall, "Rediscovering the Social Group: A Self-Categorization Theory," 1987, Basil: Blackwell press.

11. Avi Kaplan and Joanna K. Garner, "A Complex Dynamic Systems Perspective on Identity and its Development: The Dynamic Systems Model of Role Identity, Developmental Psychology vol. 53, no.11., (2017), 2036-2051.

12. Deborah Switzer and Lisa Benson, "Assessing Impact of Outreach Activity on Motivation of Undergraduate Engineering Students," paper presented at 2007 ASEE Annual Conference and Exposition (Honolulu, Hawaii: ASEE, June 2007).

13. Sara Atwood and Joshua M. Fry, "Gender Differences in Motivation to Perform K12 Outreach," paper presented at 2015 ASEE Annual Conference and Exposition (Atlanta, Georgia: ASEE, June 2015).

14. Kimberley Edginton Bigelow, "Reflections of College Students Promoting Engineering Through Biomechanical Outreach Activities Indicate Dual Benefits," paper presented at 2010 ASEE Annual Conference and Exposition (Louisville, Kentucky: ASEE, June 2010). 
15. Thalia Anagnos, Alicia Lyman-Holt, Claudia Marin-Artieda, and Ellen Momsen, "Impact of Engineering Ambassador Programs on Student Development," Journal of STEM Education: Innovations and Research, vol. 15, no. 3 (2014, October-December), pp.1419.

16. Howard L. Greene, Xi Zhan, Anika Anthony, Paul E. Post, and Andrew James Parkhurst, "Preparing Teachers and University Students to Translate Engineering Research to K8 Students in an After-School Program," paper presented at 2014 ASEE Annual Conference and Exposition (Indianapolis, Indiana: ASEE, June 2014).

17. Monique S. Ross, Trina L. Fletcher, Vishodana Thamotharan, and Atalie Garcia, "I Lead, Therefore I Am: The Impact of Student-mentor Leadership Opportunities on STEM Identity Development and Sustainability," paper presented at 2018 ASEE Annual Conference and Exposition (Salt Lake City, Utah: ASEE, June 2018).

18. Godwin, Potvin, Hazari \& Lock (2013)

19. Joanna K. Garner, Michael Alley, Christine Haas, Suzanne Sontgerath, and Avi Kaplan, "Work In Progress: Developing and Inter-Relating the Role Identities of Engineering Ambassadors through Hands-On Outreach Activities," paper presented at 2017 ASEE Annual Conference and Exposition (Columbus, Ohio: ASEE, June 2017)

20. Karen Thole, Sarah E. Zappe, Melissa Marshall, Michael Alley, and Renata Engel, "Engineering Ambassador Network: Dissemination Through an Inaugural National Workshop," paper presented at 2013 ASEE Annual Conference and Exposition (Atlanta, Georgia: ASEE, June, 2013).

21. Jan DeWaters, Susan Powers, and Mary Graham, "Partners in Engineering: Outreach Efforts Provide Holistic Engineering Education for Middle School Girls," paper presented at 2006 ASEE Annual Conference and Exposition (Chicago, Illinois: ASEE, June 2006).

22. Adam Carberry, Merredith Portsmore, and Chris Rogers, "The Effects of STOMP on Students' Attitudes and Understandings toward the Engineering Design Process,” paper presented at 2007 ASEE Annual Conference and Exposition (Honolulu, Hawaii: ASEE, June 2007).

23. Sara Jordan-Bloch, and S. Cohen, "Engagement in Practice: Infusing the STEM Pipeline Through Community Engaged Learning," paper presented at 2018 ASEE Annual Conference and Exposition (Salt Lake City, Utah: ASEE, June 2018).

24. Sara Atwood, Eli Patten, and Lisa Pruitt, "Outreach Teaching, Communication, and Interpersonal Skills Encourage Women and may Facilitate their Recruitment and Retention in the Engineering Curriculum," paper presented at ASEE Annual Conference and Exposition (Louisville, Kentucky: ASEE, June 2010).

25. Jacquelyn Sullivan and Malinda Schaefer Zarske, "The K-12 Engineering Outreach Corps: A Service-Learning Technical Elective," paper presented at 2005 ASEE Annual Conference and Exposition (Portland, Oregon: ASEE, June 2005).

26. Sara Atwood and Joshua M. Fry, "Model for Undergraduate Outreach Project Involving Community Engagement," paper presented at 2013 ASEE Annual Conference and Exposition (Atlanta, Georgia: ASEE, June 2013).

27. Christine Haas, Michael Alley, Joanna K. Garner, and Karen Thole, "Engineering Ambassadors Network (EAN): Goals, Successes, and Challenges in Growing the EAN," 
paper presented at 2016 ASEE Annual Conference and Exposition (New Orleans, Louisiana: ASEE, June 2016).

28. Zahra Shabhazi, Alexandra Emma Lehnes, Mary Ann Jacobs, and Kathleen Christal Mancuso, "Engineering Ambassadors: Bridging the Gap Between Engineering and Education Undergraduates and Middle and High School Students," paper presented at 2016 ASEE Annual Conference and Exposition (New Orleans, Louisiana: ASEE, June 2016).

29. Catherine Talbot, Michael Alley, Melissa Marshall, Sarah E. Zappe, and Joanna K. Garner, "Engineering Ambassador Network: Professional Development of the Engineering Ambassadors," paper presented at 2013 ASEE Annual Conference and Exposition (Atlanta, Georgia: ASEE, June 2013).

30. Kate Caldwell, Jessica McCoy, Lynn Albers, Althea Smith, and Elizabeth Parry, "The Impact of K-12 Outreach Program on Graduate and Undergraduate Experiences," paper presented at the 2007 ASEE Annual Conference and Exposition (Honolulu, Hawaii: June, 2007).

31. Jeffrey Scott Bates, Karen J. Krapcho, and Crystal Orantes, "How to Recruit and Retain Students Using an Engineering Ambassador Program," paper presented at 2014 ASEE Annual Conference and Exposition (Indianapolis, Indiana: ASEE, June 2014).

32. Christine Haas, Lynn S. McElholm, Sonya M. Renfro, Elizabeth S. Herkenham, Melissa Marshall, and Michael Alley, "Engineering Ambassador Network: Establishment of Successful Engineering Ambassador Programs at Four UTC Partners Schools," paper presented at 2013 ASEE Annual Conference and Exposition (Atlanta, Georgia: ASEE, June 2013).

33. Christian Scherrer, "Improved Retention and Other Impacts Benefiting Engineering Technology Undergraduates Involved in High School Outreach," paper presented at 2013 ASEE Annual Conference and Exposition (Atlanta, Georgia: ASEE, June 2013).

34. Shannon Ciston, Ellen Worsdall, and Jessica Swenson, "Summer Technology and Engineering Program: An Outreach Program Benefitting Women Engineering Students From Middle School Through Graduate School," paper presented at 2010 ASEE Annual Conference and Exposition (Louisville, Kentucky: ASEE, June 2010).

35. Noah Salzman and Johanes Strobel, "Motivations and Benefits for College Students Serving as Mentors in a High School Robotics Competition," paper presented at 2011 ASEE Annual Conference and Exposition (Vancouver, British Columbia: ASEE, June, 2011).

36. Bethany Bowling, Maureen Doyle, Jennifer Taylor, and Alison Antes, "Professionalizing the Role of Peer Leaders in STEM," paper presented at 2015 ASEE Annual Conference and Exposition (Seattle, Washington: ASEE, June 2015).

37. Ari Epstein, Beverly Mire, Trent Ramsey, Karen Gareis, Emily Davidson, Elizabeth Jones, Michelle Slosberg, and Rafael Bras, "Terrascope Youth Radio: Engaging Urban Teens in a Unique University-Community Partnership," paper presented at 2010 ASEE Annual Conference and Exposition (Louisville, Kentucky: ASEE, June 2010).

38. Joanna Garner, Michael Alley, Christine Haas, and Avi Kaplan, "From Undergraduates to Engineering Ambassadors: The Impact of Engineering Ambassadors Network Training," paper presented at 2016 ASEE Annual Conference and Exposition (New Orleans, Louisiana: ASEE, June 2016). 
39. Rachel Jannette McFalls, Carla Danielle Grimes, M. Jean Mohammadi-Aragh, Rani W. Sullivan, and James Warncok, 'Undergraduate Facilitators' Perspectives of Engineering Summer Programs," paper presented at 2015 ASEE Annual Conference and Exposition (Seattle, Washington: ASEE, June, 2015).

40. Hadil Mustafa, and Shelby Ann Freese, "Impact of 'Imagineer Day,' an Outreach Program, on K_8 Girls and Women in Engineering," paper presented at 2018 ASEE Annual Conference and Exposition (Salt Lake City, Utah: ASEE, June, 2018).

41. Suzanne A. Olds, Chirag D. Patel, Bugrahan Yalvac, David E. Kanter and Neha Goel, "Developing a Standards-based K-12 Engineering Curricula Through Partnership with University Students and Industry," paper presented at 2004 ASEE Annual Conference and Exposition (Portland, Oregon: ASEE, June, 2004).

42. Suzanne A. Olds, David E. Kanter, Amanda Knudson, and Shruti B. Mehta, "Designing and Outreach Project that Trains Both Future Faculty and Future Engineers," paper presented at 2003 ASEE Annual Conference and Exposition (Nashville, Tennessee: ASEE, June 2003).

43. Luz M. Rivas and D. Olmsted, "Be A Scientist: Family Science for Minorities," paper presented at 2013 ASEE Annual Conference and Exposition (Atlanta, Georgia: June, 2013).

44. Lynn Albers, Althea Smith, Kate Caldwell, Jessica McCoy, Laura Bottomly, and Elizabeth Parry, "The Impact of Out-of-School (OST) Math and Science Clubs on elementary and Middle School Students, Teachers, Schools and the Undergraduate and Graduate Fellows that Facilitate Them," paper presented at the 2008 ASEE Annual Conference and Exposition (Pittsburgh, Pennsylvania: June, 2008).

45. Joshua M. Frey, and Sara A. Atwood, "Model for Undergraduate Outreach Project Involving Community Engagement," paper presented at 2013 ASEE Annual Conference and Exposition (Atlanta, Georgia: ASEE, June, 2013).

46. Jayser Jahan, Beena Sukumaran, Linda M. Head, and Zenida O. Keil, "AWE: An Outreach Workshop for Middle School Girls," paper presented at 2012 ASEE Annual Conference and Exposition (San Antonio, Texas: ASEE, June 2012).

47. Sarah M. Brown, and Lauren Thomas, "Technical Outreach Community Help: Initial Results," paper presented at 2011 ASEE Annual Conference and Exposition (Vancouver, British Columbia: ASEE, June 2011).

48. Howard L. Greene, Paul E. Post, and Lisa Abrams, "Engineering Ambassador Program Connects High School Students with University Students and Career Engineers in their Communities," paper presented at 2015 ASEE Annual Conference and Exposition (Seattle, Washington: ASEE, June 2015).

49. Ayyana Chakravartula, Barbara Ando, Cheng Li, Shikha Gupta, and Lisa Pruitt, "Undergraduate Students Teaching Children: K-8 Outreach Within the Core Engineering Curriculum," paper presented at 2006 ASEE Annual Conference and Exposition (Chicago, Illinois: ASEE, June 2006).

50. Cristina D. Pomales-Garcia, Oscar Marcelo Suarez, Agnes M. Padovani, and Jaquelina E. Alvarez, "A Successful Pre-College Nanotechnology Experience for Low-Income Students (Evaluation)," paper presented at 2018 ASEE Annual Conference and Exposition (Salt Lake City, Utah: ASEE, June 2018). 
51. Adrienne Steele and Waggenspack, "STEP-ing UP: Building a Successful Student Leadership Program," paper presented at 2018 ASEE Annual Conference and Exposition (Salt Lake City, Utah: ASEE, June 2018).

52. Michael Alley, Christine Haas, Joanna K. Garner, and Karen Thole, "Engineering Ambassador Network: Progress in 2014 on Creating a National Network of Ambassadors," paper presented at 2015 ASEE Annual Conference and Exposition (Seattle, Washington: ASEE, June, 2015). 